\title{
Hadronic Molecular States Composed of Heavy Flavor Baryons
}

\author{
Ning L: \\ Department of Physics and State Key Laboratory of Nuclear Physics and Technology \\ Peking University, Beijing 100871, China \\ Shi-Lin Zhu \\ Department of Physics and State Key Laboratory of Nuclear Physics and Technology \\ and Center of High Energy Physics, Peking University, Beijing 100871, China
}

\begin{abstract}
We investigate the possible molecules composed of two heavy flavor baryons such as " $A_{Q} B_{Q}$ " $(Q=b, c)$ within the one-pion-exchange model (OPE). Our results indicate that the long-range $\pi$ exchange force is strong enough to form molecules such as $\left[\Sigma_{Q} \Xi_{Q}^{\prime}\right]_{S=1}^{I=1 / 2}(Q=b, c),\left[\Sigma_{Q} \Lambda_{Q}\right]_{S=1}^{I=1}(Q=b, c),\left[\Sigma_{b} \Xi_{b}^{\prime}\right]_{S=1}^{I=3 / 2}$ and $\left[\Xi_{b} \Xi_{b}^{\prime}\right]_{S=1}^{I=0}$ where the S-D mixing plays an important role. In contrast, the $\pi$ exchange does not form the spin-singlet $A_{Q} B_{Q}$ bound states. If we consider the heavier scalar and vector meson exchanges as well as the pion exchange, some loosely bound spin-singlet $\mathrm{S}$-wave states appear while results of the spin-triplet $A_{Q} B_{Q}$ system do not change significantly, which implies the pion exchange plays an dominant role in forming the spin-triplet molecules. Moreover, we perform an extensive coupled channel analysis of the $\Lambda_{Q} \Lambda_{Q}$ system within the OPE and oneboson-exchange (OBE) framework and find that there exist loosely bound states of $\Lambda_{Q} \Lambda_{Q}(Q=b, c)$ with quantum numbers $I\left(J^{P}\right)=0\left(0^{+}\right), O\left(0^{-}\right)$and $0\left(1^{-}\right)$. The binding solutions of $\Lambda_{Q} \Lambda_{Q}$ system mainly come from the coupled-channel effect in the flavor space. Besides the OPE force, the medium- and short-range attractive force also plays a significant role in the formation of the loosely bound $\Lambda_{c} \Lambda_{c}$ and $\Lambda_{b} \Lambda_{b}$ states. Once produced, they will be very stable because such a system decays via weak interaction with a very long lifetime around $10^{-13} \sim 10^{-12} \mathrm{~s}$.

PACS numbers: 12.39.Pn, 14.20.-c, 12.40.Yx
\end{abstract}

\section{INTRODUCTION}

In the past few years, many exotic charmonium-like states have been reported by the Belle, BARBAR, CDF and D0 collaborations, such as $X(3872)[1], X(4160)$ [2], $Y(4260)$ [3], and $Z^{+}(4430)$ [4]. Recently, the BELLE Collaboration observed two charged bottomonium-like resonances $Z_{b}(10610)$ and $Z_{b}(10650)$ in the hidden-bottom decay channels $\pi^{ \pm} \Upsilon(n S)(n=1,2,3)$ and $\pi^{ \pm} h_{b}(m P)(m=1,2)$ of $\Upsilon(5 S)$ [5]. Since many of these states do not fit into the conventional $q \bar{q}$ picture in the quark model easily, how to interpret these "exotic" states becomes a challenging problem. The prominent feature of these states is that they are near the threshold of two charmed or bottomed mesons. For example, $X(3872)$ lies close to the threshold of $D^{0} \bar{D}^{* 0}$ while $Z_{b}(10610)$ and $Z_{b}(10650)$ are near the threshold of $B \bar{B}^{*}$ and $B^{*} \bar{B}^{*}$ respectively. Inspired by this striking feature of these exotic states, many physicists attempted to interpret them as hadronic molecules composed of heavy mesons.

A hadronic molecular state is a loosely bound state of hadrons. Voloshin and Okun began to investigate the existence of the bound states composed of charmed meson and antimeson [6]. De Rujula et al proposed $\psi(4040)$ might be a $D^{*} \bar{D}^{*}$ molecular state [7]. Törnqvist explored the possible deuteron-like meson-antimeson bound states with the pion exchange potential [8, 9]. Liu et al. investigated the possible molecular states composed of heavy mesons within the framework of the one-boson-exchange model (OBE) [10]. Ding et al. also gave a dynamic study of meson-meson molecular states with the one-boson-exchange model at the quark level [11]. Sun et al. interpreted the newly observed $Z_{b}(10610)$ and $Z_{b}(10650)$ as $B \bar{B}^{*}$ and $B^{*} \bar{B}^{*}$ molecular states respectively [12].

Actually, the idea of the loosely bound molecular states is not new in nuclear physics. It's well-known that the deuteron is a very loosely bound state composed of a proton and neutron. The interaction between the proton and neutron comes from the color-singlet meson exchange. Besides the long-range attraction from the pion exchange, the S-D mixing, the medium-range attraction from the correlated two-pion exchange (or in the form of the sigma meson exchange), and the short-range interaction in terms of the vector meson exchange combine to form the loosely bound deuteron.

It's quite natural to extend the same formalism to the heavy baryon sector. Since the heavy baryon contains a charm or bottom quark, its large mass reduces the kinetic energy and helps the formation of the bound states. Fröemel et al investigated the bound states composed of heavy hyperon and nucleon by rescaling the nucleon-nucleon potential in [13]. Juliá-Diaz et al explored

*Electronic address: leening@pku.edu.cn

${ }^{\dagger}$ Electronic address: zhusl@pku.edu.cn 
the bound states composed of double-charmed hyperons [14]. In our previous work [19], we performed a study of the systems $\Lambda_{c} \Lambda_{c}\left(\bar{\Lambda}_{c}\right), \Xi_{c} \Xi_{c}\left(\bar{\Xi}_{c}\right), \Sigma_{c} \Sigma_{c}\left(\bar{\Sigma}_{c}\right), \Xi_{c}^{\prime} \Xi_{c}^{\prime}\left(\bar{\Xi}_{c}^{\prime}\right)$ and $\Omega_{c} \Omega_{c}\left(\bar{\Omega}_{c}\right)$.

In the present work we shall study the systems with two different heavy flavor baryons. For simplicity, we denote the systems with two same baryons as " $A_{Q} A_{Q}$ " and the systems with two different baryons as " $A_{Q} B_{Q}$ ". The difference between the two systems is that the " $A_{Q} B_{Q}$ " system contains the contributions coming from the $K^{ \pm}, K^{0}, \bar{K}^{0}, K^{* \pm}, K^{* 0}$ and $\bar{K}^{* 0}$ exchange while the " $A_{Q} A_{Q}$ " system does not.

Among the possible loosely bound states composed of a pair of heavy baryons, the $\Lambda_{Q} \Lambda_{Q}(Q=b, c)$ system is particularly interesting since it is the heavy analogue of the well-known H dibaryon. Since it was proposed by Jaffe in Ref. [15], there have been lots of theoretical and experimental efforts. Recent investigations include the Lattice QCD calculation [16], calculations using the chiral effective field theory [17] and the quark model [18]. In this work, we shall perform an extensive coupled channel analysis of the $\Lambda_{Q} \Lambda_{Q}(Q=b, c)$ system and investigate the role of the OPE and sigma/omega/rho meson exchange in the formation of the possible loosely bound state.

This work is organized as follows. After the introduction, we present the formalism in Section $\prod$ which contains the Lagrangians, the coupling constants and the effective interaction potentials. The formalism for the coupled channel analysis of the $\Lambda_{Q} \Lambda_{Q}$ system is given in Section IIII In Sections IV and $\nabla$ we show the numerical results for " $A_{Q} B_{Q}$ " and $\Lambda_{Q} \Lambda_{Q}$ systems, respectively. The last section VI is a brief summary. Some useful formulae and functions are given in the Appendix. As a byproduct, we also collect the numerical results for the loosely bound states composed of a pair of heavy baryon and anti-baryon in the Appendix.

\section{FORMALISM}

\section{A. The lagrangian}

The heavy flavor baryon contains a charm or bottom quark and a diquark (two light quarks). In the heavy quark limit $\left(m_{Q} \rightarrow \infty\right)$, the charm or bottom quark can be viewed as a static color source. The SU(3) flavor symmetry of the baryon is determined by the diquark. The heavy flavor baryons can be classified in terms of the symmetry of the diquark. The symmetric one belongs to the 6-representation while the antisymmetric one belongs to the $\overline{3}$-representation. On the other hand, the spin of the diquark is either 0 or 1 which is antisymmetric or symmetric under the exchange of its two light quark spins. The baryon is a fermion system. Its total wave function should be antisymmetric under the exchange of its two light quarks. Therefore, the spin and the flavor of the diquark are correlated with each other. Taking the color wave function into account, the diquark in the 6-representation should be spin-triplet while the one in the $\overline{3}$-representation should be spin-singlet. The spin of the baryon in the 6-representation is either $\frac{1}{2}$ or $\frac{3}{2}$ while the spin of the baryon in the $\overline{3}$-representation is only $\frac{1}{2}$.

In the following, we follow the notations in Ref. [23] and list the heavy flavor baryon matrices and the exchanged meson matrices. The heavy flavor baryons are

$$
B_{6}=\left(\begin{array}{ccc}
\Sigma_{Q}^{+1} & \frac{1}{\sqrt{2}} \Sigma_{Q}^{0} & \frac{1}{\sqrt{2}} \Xi_{Q}^{\prime+\frac{1}{2}} \\
\frac{1}{\sqrt{2}} \Sigma_{Q}^{0} & \Sigma_{Q}^{-1} & \frac{1}{\sqrt{2}} \Xi_{Q}^{\prime-\frac{1}{2}} \\
\frac{1}{\sqrt{2}} \Xi_{Q}^{\prime+\frac{1}{2}} & \frac{1}{\sqrt{2}} \Xi_{Q}^{\prime-\frac{1}{2}} & \Omega_{Q}
\end{array}\right), \quad B_{6}^{*}=\left(\begin{array}{ccc}
\Sigma_{Q}^{*+1} & \frac{1}{\sqrt{2}} \Sigma_{Q}^{* 0} & \frac{1}{\sqrt{2}} \Xi_{Q}^{*^{\prime}+\frac{1}{2}} \\
\frac{1}{\sqrt{2}} \Sigma_{Q}^{* 0} & \Sigma_{Q}^{*-1} & \frac{1}{\sqrt{2}} \Xi_{Q}^{*^{\prime}-\frac{1}{2}} \\
\frac{1}{\sqrt{2}} \Xi_{Q}^{*^{\prime}+\frac{1}{2}} & \frac{1}{\sqrt{2}} \Xi_{Q}^{*^{\prime}-\frac{1}{2}} & \Omega_{Q}^{*}
\end{array}\right), \quad B_{\overline{3}}=\left(\begin{array}{ccc}
0 & \Lambda_{Q} & \Xi_{Q}^{+\frac{1}{2}} \\
-\Lambda_{Q} & 0 & \Xi_{Q}^{-\frac{1}{2}} \\
-\Xi_{Q}^{+\frac{1}{2}} & -\Xi_{Q}^{-\frac{1}{2}} & 0
\end{array}\right) .
$$

The spin $\frac{3}{2}$ baryon is marked with $*$. The superscript is the third component of its isospin. $Q=b$ or $c$ denotes the corresponding heavy quark. The exchanged bosons are

$$
\mathcal{M}=\left(\begin{array}{ccc}
\frac{\pi^{0}}{\sqrt{2}}+\frac{\eta}{\sqrt{6}} & \pi^{+} & K^{+} \\
\pi^{-} & -\frac{\pi^{0}}{\sqrt{2}}+\frac{\eta}{\sqrt{6}} & K^{0} \\
K^{-} & \bar{K}^{0} & -\frac{2}{\sqrt{6}} \eta
\end{array}\right), \quad \mathcal{V}^{\mu}=\left(\begin{array}{ccc}
\frac{\rho^{0}}{\sqrt{2}}+\frac{\omega}{\sqrt{2}} & \rho^{+} & K^{*+} \\
\rho^{-} & -\frac{\rho^{0}}{\sqrt{2}}+\frac{\omega}{\sqrt{2}} & K^{* 0} \\
K^{*-} & \bar{K}^{* 0} & \phi
\end{array}\right)^{\mu} .
$$

The exchanged bosons include the pseudoscalar and vector mesons given in Eq. (2) and the scalar meson $\sigma$. The lagrangians built under the SU(3)-flavor symmetry read as

$$
\mathcal{L}=\mathcal{L}_{p h h}+\mathcal{L}_{v h h}+\mathcal{L}_{\sigma h h},
$$

where

$$
\begin{aligned}
& \mathcal{L}_{p h h}=g_{p B_{6} B_{6}} \operatorname{Tr}\left[\bar{B}_{6} i \gamma_{5} \mathcal{M} B_{6}\right]+g_{p B_{\overline{3}} B_{\overline{3}}} \operatorname{Tr}\left[\bar{B}_{\overline{3}} i \gamma_{5} \mathcal{M} B_{\overline{3}}\right]+\left\{g_{p B_{6} B_{\overline{3}}} \operatorname{Tr}\left[\bar{B}_{6} i \gamma_{5} \mathcal{M} B_{\overline{3}}\right]+\text { h.c. }\right\}, \\
& \mathcal{L}_{v h h}=g_{v B_{6} B_{6}} \operatorname{Tr}\left[\bar{B}_{6} \gamma_{\mu} \mathcal{V}^{\mu} B_{6}\right]+\frac{f_{v B_{6} B_{6}}}{2 m_{6}} \operatorname{Tr}\left[\bar{B}_{6} \sigma_{\mu \nu} \partial^{\mu} \mathcal{V}^{\nu} B_{6}\right]+g_{v B_{\overline{3}} B_{\overline{3}}} \operatorname{Tr}\left[\bar{B}_{\overline{3}} \gamma_{\mu} \mathcal{V}^{\mu} B_{\overline{3}}\right]+\frac{f_{v B_{\overline{3}} B_{\overline{3}}}}{2 m_{\overline{3}}} \operatorname{Tr}\left[\bar{B}_{\overline{3}} \sigma_{\mu \nu} \partial^{\mu} \mathcal{V}^{\nu} B_{\overline{3}}\right]
\end{aligned}
$$




$$
\begin{aligned}
&+\left\{g_{v B_{6} B_{\overline{3}}} \operatorname{Tr}\left[\bar{B}_{6} \gamma_{\mu} \mathcal{V}^{\mu} B_{\overline{3}}\right]+\frac{f_{v B_{6} B_{\overline{3}}}}{2 \sqrt{m_{6} m_{\overline{3}}}} \operatorname{Tr}\left[\bar{B}_{6} \sigma_{\mu \nu} \partial^{\mu} \mathcal{V}^{\nu} B_{\overline{3}}\right]+\text { h.c. }\right\}, \\
& \mathcal{L}_{\sigma h h}=g_{\sigma B_{6} B_{6}} \operatorname{Tr}\left[\bar{B}_{6} \sigma B_{6}\right]+g_{\sigma B_{\overline{3}} B_{\overline{3}}} \operatorname{Tr}\left[\bar{B}_{\overline{3}} \sigma B_{\overline{3}}\right] .
\end{aligned}
$$

$\mathcal{M}$ and $\mathcal{V}^{\mu}$ are the exchanged pseudoscalar and vector meson matrices, respectively, which are given in Eq. (2). $B_{6}$ and $B_{\overline{3}}$ are the heavy baryon matrices shown in Eq. (1). $m_{6}$ and $m_{\overline{3}}$ are the masses of the heavy baryons belonging to the 6-representation and $\overline{3}$-representation, respectively. " $g_{p B_{6} B_{6}}, g_{v B_{6} B_{6}}, \ldots$ " are the coupling constants.

\section{B. Coupling Constants}

We derive the coupling constants in Eqs. (3) 5) from those between the nucleon and the light meson within the Quark Model $(\mathrm{QM})$. For the vector meson exchange, we adopt the lagrangian without the anomalous magnetic term at the quark level as in Ref. [24]. One can refer to Ref. [19] for the specific expressions of the couplings at the quark level in terms of those between the nucleon and the exchanged mesons. We list the coupling constants we need below and collect their numerical results in Table [

$$
\begin{gathered}
\text { pseudoscalar exchange, } \quad g_{p B_{6} B_{6}}=\frac{4 \sqrt{2}}{5} g_{\pi N N} \frac{m_{i}+m_{f}}{2 m_{N}}, \quad g_{p B_{6} B_{3}}=-\frac{2 \sqrt{3}}{5} g_{\pi N N} \frac{m_{i}+m_{f}}{2 m_{N}}, \quad g_{p B_{3} B_{3}}=0 . \\
\text { scalar exchange, } \quad g_{\sigma B_{6} B_{6}}=\frac{2}{3} g_{\sigma N N}, \quad g_{\sigma B_{3} B_{3}}=\frac{1}{3} g_{\sigma N N} . \\
\text { vector exchange, } \quad g_{v B_{6} B_{6}}=2 \sqrt{2} g_{\rho N N}, \quad f_{v B_{6} B_{6}}=\frac{4 \sqrt{2}}{5}\left(g_{\rho N N}+f_{\rho N N}\right) \frac{\sqrt{m_{i} m_{f}}}{m_{N}}-2 \sqrt{2} g_{\rho N N}, \\
g_{v B_{3} B_{3}}=\sqrt{2} g_{\rho N N}, \quad f_{v B_{3} B_{3}}=-\sqrt{2} g_{\rho N N}, \\
g_{v B_{6} B_{3}}=0, \quad f_{v B_{6} B_{3}}=-\frac{2 \sqrt{3}}{5}\left(g_{\rho N N}+f_{\rho N N}\right) \frac{\sqrt{m_{i} m_{f}}}{m_{N}} .
\end{gathered}
$$

In the above expressions, $g_{\pi N N}, g_{\sigma N N}, g_{\rho N N}$ and $f_{\rho N N}$ are the coupling constants between the nucleon and the exchanged mesons. Their numerical values are known quite well. $m_{N}$ is the mass of the nucleon. $m_{i}$ and $m_{f}$ are the masses of the ingoing and outgoing baryons, respectively. From Eqs. (68), the value of the same coupling constant is slightly different for different systems if one takes into account the mass difference of the baryons of the same representation. For example, $g_{p B_{6} B_{6}}=\frac{4 \sqrt{2}}{5} g_{\pi N N} \frac{m_{\Sigma_{c}}+m_{\Sigma_{c}}}{2 m_{N}}$ for the

interaction vertex $\Sigma_{c} \Sigma_{c} \pi$ while $g_{p B_{6} B_{6}}=\frac{4 \sqrt{2}}{5} g_{\pi N N} \frac{m_{\Xi_{c}^{\prime}}+m_{\Xi_{c}^{\prime}}}{2 m_{N}}$ for the $\Xi_{c}^{\prime} \Xi_{c}^{\prime} \pi$ vertex. The masses of the baryons and exchanged mesons are summarized in Table $\left[\right.$ The values of the coupling constants are [24-26] : $g_{\pi N N}^{2} / 4 \pi=13.6, g_{\eta N N}=0.4, g_{\sigma N N}^{2} / 4 \pi=5.69$, $g_{\rho N N}^{2} / 4 \pi=0.84, f_{\rho N N} / g_{\rho N N}=\kappa_{\rho}=6.1, g_{\omega N N}^{2} / 4 \pi=20.0, f_{\omega N N} / g_{\omega N N}=\kappa_{\omega}=0$. In our case, we assume the SU(3) symmetry. Therefore we need only three couplings for the pseudoscalar, scalar and vector meson exchange respectively. We adopt the three independent ones as $g_{\pi N N}, g_{\sigma N N}$ and $g_{\rho N N}$, since they do not vary much among different models. Generally speaking, the physical results of the loosely bound deuteron system are very sensitive to the vector meson coupling constants. The recently proposed renormalization approach, which uses a regularized boundary condition, can decrease the dependence on the coupling constants [27].

\section{Effective Potential}

Applying the lagrangians in Eqs.(3) 5) one can derive the effective interaction potential in the momentum space. Given the hadrons are not fundamental particles, we employ a monopole form factor at each vertex to roughly describe the structure effect of the baryon

$$
\mathcal{F}(Q)=\frac{\Lambda^{2}-m_{e x}^{2}}{\Lambda^{2}-Q^{2}}=\frac{\Lambda^{2}-m_{e x}^{2}}{\lambda^{2}+Q^{2}},
$$

where $\Lambda$ is the cutoff parameter by which we can regulate the exchanged momentum. $m_{e x}$ and $Q$ are the mass and four momentum of the exchanged meson, respectively, and $\lambda^{2}=\Lambda^{2}-Q_{0}^{2}$. Making the Fourier transformation,

$$
\mathcal{V}(r)=\frac{1}{(2 \pi)^{3}} \int d Q^{3} e^{i Q \cdot r} \mathcal{V}(Q) \mathcal{F}^{2}(Q),
$$


TABLE I: The numerical results of the coupling constants. " $\times$ ” means such a vertex does not exist.

\begin{tabular}{|c|c|c|c|c|c|c|c|c|}
\hline \multirow[t]{2}{*}{ Vertex } & \multicolumn{4}{|c|}{$\mathrm{Q}=\mathrm{c}$} & \multicolumn{4}{|c|}{$\mathrm{Q}=\mathrm{b}$} \\
\hline & $g_{p B_{6} B_{6}}$ & $g_{\sigma B_{6} B_{6}}$ & $g_{v B_{6} B_{6}}$ & $f_{v B_{6} B_{6}}$ & $g_{p B_{6} B_{6}}$ & $g_{\sigma B_{6} B_{6}}$ & $g_{v B_{6} B_{6}}$ & $f_{v B_{6} B_{6}}$ \\
\hline$\Sigma_{Q} \Sigma_{Q}(p, \sigma, v)$ & 38.69 & 5.64 & 9.19 & 59.08 & 91.64 & 5.64 & 9.19 & 152.51 \\
\hline$\Sigma_{q} \Xi_{Q}^{\prime}(p, \sigma, v)$ & 39.65 & $x$ & 9.19 & 60.76 & 92.66 & $x$ & 9.19 & 154.30 \\
\hline$\Sigma_{Q} \Omega_{Q}(p, \sigma, v)$ & 40.61 & $\times$ & 9.19 & 62.39 & 93.68 & $\times$ & 9.19 & 156.08 \\
\hline$\Xi_{Q}^{\prime} \Xi_{Q}^{\prime}(p, \sigma, v)$ & 40.62 & 5.64 & 9.19 & 62.48 & 93.68 & 5.64 & 9.19 & 156.18 \\
\hline$\Xi_{Q}^{\prime} \Omega_{Q}(p, \sigma, v)$ & 41.58 & $\times$ & 9.19 & 64.15 & 94.71 & $\times$ & 9.19 & 157.91 \\
\hline \multirow[t]{2}{*}{$\Omega_{Q} \Omega_{Q}(p, \sigma, v)$} & 42.53 & 5.64 & 9.19 & 65.86 & 95.73 & 5.64 & 9.19 & 159.73 \\
\hline & $g_{p B_{6} B_{\overline{3}}}$ & $g_{\sigma B_{6} B_{\overline{3}}}$ & $g_{v B_{6} B_{\overline{3}}}$ & $f_{v B_{6} B_{\overline{3}}}$ & $g_{p B_{6} B_{\overline{3}}}$ & $g_{\sigma B_{6} B_{\overline{3}}}$ & $g_{v B_{6} B_{\overline{3}}}$ & $f_{v B_{6} B_{\overline{3}}}$ \\
\hline$\Sigma_{Q} \Lambda_{Q}(p, \sigma, v)$ & -22.89 & $\times$ & 0 & -40.36 & -55.19 & $\times$ & 0 & -97.37 \\
\hline$\Sigma_{Q} \Xi_{Q}(p, \sigma, v)$ & -23.77 & $\times$ & 0 & -41.95 & -56.01 & $\times$ & 0 & -98.84 \\
\hline$\Xi_{Q}^{\prime} \Lambda_{Q}(p, \sigma, v)$ & -23.48 & $\times$ & 0 & -41.35 & -55.82 & $\times$ & 0 & -98.46 \\
\hline$\Xi_{Q}^{\prime} \Xi_{Q}(p, \sigma, v)$ & -24.36 & $\times$ & 0 & -42.98 & -56.64 & $x$ & 0 & -99.94 \\
\hline \multirow[t]{2}{*}{$\Omega_{Q} \Xi_{Q}(p, \sigma, v)$} & -24.95 & $\times$ & 0 & -43.98 & -57.27 & $\times$ & 0 & -101.02 \\
\hline & $g_{p B_{\overline{3}} B_{\overline{3}}}$ & $g_{\sigma B_{\overline{3}} B_{\overline{3}}}$ & $g_{v B_{\overline{3}} B_{\overline{3}}}$ & $f_{v B_{\overline{3}} B_{\overline{3}}}$ & $g_{p B_{\overline{3}} B_{\overline{3}}}$ & $g_{\sigma B_{\overline{3}} B_{\overline{3}}}$ & $g_{v B_{\overline{3}} B_{\overline{3}}}$ & $f_{v B_{\overline{3}} B_{\overline{3}}}$ \\
\hline$\Lambda_{Q} \Lambda_{Q}(p, \sigma, v)$ & 0 & 2.82 & 4.59 & -4.59 & 0 & 2.82 & 4.59 & -4.59 \\
\hline$\Lambda_{Q} \Xi_{Q}(p, \sigma, v)$ & 0 & $\times$ & 4.59 & -4.59 & 0 & $\times$ & 4.59 & -4.59 \\
\hline$\Xi_{Q} \Xi_{Q}(p, \sigma, v)$ & 0 & 2.82 & 4.59 & -4.59 & 0 & 2.82 & 4.59 & -4.59 \\
\hline
\end{tabular}

one obtains the effective interaction potential in the coordinate space, which are given below. One can refer to Appendix VII A for some formulae. Since the hadronic molecule is a loosely bound state, the hadrons are not expected to be very close to each other. We neglect the contact interaction piece $\delta(r)$ in the potential. The detailed information of the delta term is given in the Appendix.

We expand the effective interaction potential in terms of $\frac{1}{m}$ ( $\mathrm{m}$ is the heavy baryon mass) up to order of $\frac{1}{m^{2}}$. We also adopt the approximation $1 / m_{A}^{2} \sim 1 / m_{B}^{2} \sim 1 /\left(m_{A} m_{B}\right)$ due to the large masses $\left(m_{A}, m_{B}\right)$ of the heavy baryons. The effective potentials have four terms: the central potential term $\mathcal{V}_{C}$, the spin-spin term $\mathcal{V}_{S S}$, the spin-orbit term $\mathcal{V}_{L S}$ and the tensor term $\mathcal{V}_{T}$.

$$
\begin{aligned}
\mathcal{V}^{p}(r ; n, \alpha) & =\mathcal{V}_{S S}^{p}(r ; n, \alpha)+\mathcal{V}_{T}^{p}(r ; n, \alpha), \\
\mathcal{V}^{s}(r ; n=1, \sigma) & =\mathcal{V}_{C}^{s}(r ; n=1, \sigma)+\mathcal{V}_{L S}^{s}(r ; n=1, \sigma), \\
\mathcal{V}^{v}(r ; n, \beta) & =\mathcal{V}_{C}^{v}(r ; n, \beta)+\mathcal{V}_{L S}^{v}(r ; n, \beta)+\mathcal{V}_{S S}^{v}(r ; n, \beta)+\mathcal{V}_{T}^{v}(r ; n, \beta),
\end{aligned}
$$

where the superscripts, $p, s$ and $v$, denote the pseudoscalar, scalar and vector meson exchange, respectively. $\alpha=\pi, \eta, K^{ \pm}, K^{0}$, $\bar{K}^{0}$ and $\beta=\omega, \rho, \phi, K^{* \pm}, K^{* 0}, \bar{K}^{* 0} . n=1,2$ denotes the direct and cross diagrams, respectively. For the scalar exchange, " $n=1$ " means that the $\sigma$ exchange only occurs in the direct diagram. The specific expressions read

$$
\begin{aligned}
\text { pseudoscalar exchange, } \quad \mathcal{V}_{S S}^{p}(r ; n, \alpha) & =C_{n, \alpha}^{p} \frac{g_{1 p} g_{2 p}}{4 \pi} \frac{u_{\alpha}^{3}}{12 m_{A} m_{B}} H_{0}\left(\Lambda, m_{\alpha}, r\right) \sigma_{A} \cdot \sigma_{B}, \\
\mathcal{V}_{T}^{p}(r ; n, \alpha) & =C_{n, \alpha}^{p} \frac{g_{1 p} g_{2 p}}{4 \pi} \frac{u_{\alpha}^{3}}{12 m_{A} m_{B}} H_{3}\left(\Lambda, m_{\alpha}, r\right) S_{A B}(\hat{r}),
\end{aligned}
$$

when $u_{\alpha}^{2}=m_{\alpha}^{2}-\left(m_{f}-m_{i}\right)^{2}<0$, they change into,

$$
\begin{aligned}
\mathcal{V}_{S S}^{p}(r ; n, \alpha) & =C_{n, \alpha}^{p} \frac{g_{1 p} g_{2 p}}{4 \pi} \frac{\theta_{\alpha}^{3}}{12 m_{A} m_{B}} M_{0}\left(\Lambda, m_{\alpha}, r\right) \sigma_{A} \cdot \sigma_{B}, \\
\mathcal{V}_{T}^{p}(r ; n, \alpha) & =C_{n, \alpha}^{p} \frac{g_{1 p} g_{2 p}}{4 \pi} \frac{\theta_{\alpha}^{3}}{12 m_{A} m_{B}} M_{3}\left(\Lambda, m_{\alpha}, r\right) S_{A B}(\hat{r}),
\end{aligned}
$$


TABLE II: The masses of the relevant mesons and baryons [28]. The bottomed baryons $\Sigma_{b}^{0}, \Xi_{b}^{\prime 0}$ and $\Xi_{b}^{\prime-}$ (marked with $*$ ) have not been observed experimentally. Their masses are fixed to be: $m_{\Sigma_{b}^{0}}=\left[m_{\Sigma_{b}^{+}}+m_{\Sigma_{b}^{-}}\right] / 2$ and $m_{\Xi_{b}^{\prime 0}}=m_{\Xi_{b}^{\prime}}=\left[m_{\Sigma_{b}}+m_{\Omega_{b}}\right] / 2$.

\begin{tabular}{cccccc}
\hline meson & mass $(\mathrm{MeV})$ & baryon & mass $(\mathrm{MeV})$ & baryon & mass(MeV) \\
\hline$\pi^{ \pm}$ & 139.57 & $\Lambda_{c}^{+}$ & 2286.5 & $\Lambda_{b}^{0}$ & 5620.2 \\
$\pi^{0}$ & 134.98 & $\Xi_{c}^{+}$ & 2467.9 & $\Xi_{b}^{0}$ & 5790.5 \\
$\eta$ & 547.85 & $\Xi_{c}^{0}$ & 2471.0 & $\Xi_{b}^{-}$ & 5790.5 \\
$\rho$ & 775.49 & $\Sigma_{c}^{++}$ & 2454.02 & $\Sigma_{b}^{+}$ & 5807.8 \\
$\omega$ & 782.65 & $\Sigma_{c}^{+}$ & 2452.9 & ${ }^{*} \Sigma_{b}^{0}$ & 5811.5 \\
$\phi$ & 1019.46 & $\Sigma_{c}^{0}$ & 2453.8 & $\Sigma_{b}^{-}$ & 5815.2 \\
$K^{ \pm}$ & 493.68 & $\Xi_{c}^{\prime+}$ & 2575.7 & ${ }^{*} \Xi_{b}^{\prime 0}$ & 5941.3 \\
$K^{0}$ & 497.61 & $\Xi_{c}^{\prime 0}$ & 2578.0 & ${ }^{*} \Xi_{b}^{\prime-}$ & 5941.3 \\
$K^{* \pm}$ & 891.66 & $\Omega_{c}^{0}$ & 2697.5 & $\Omega_{b}^{-}$ & 6071.0 \\
$K^{* 0}$ & 895.94 & & & & \\
$\sigma$ & 600 & & & & \\
\hline
\end{tabular}
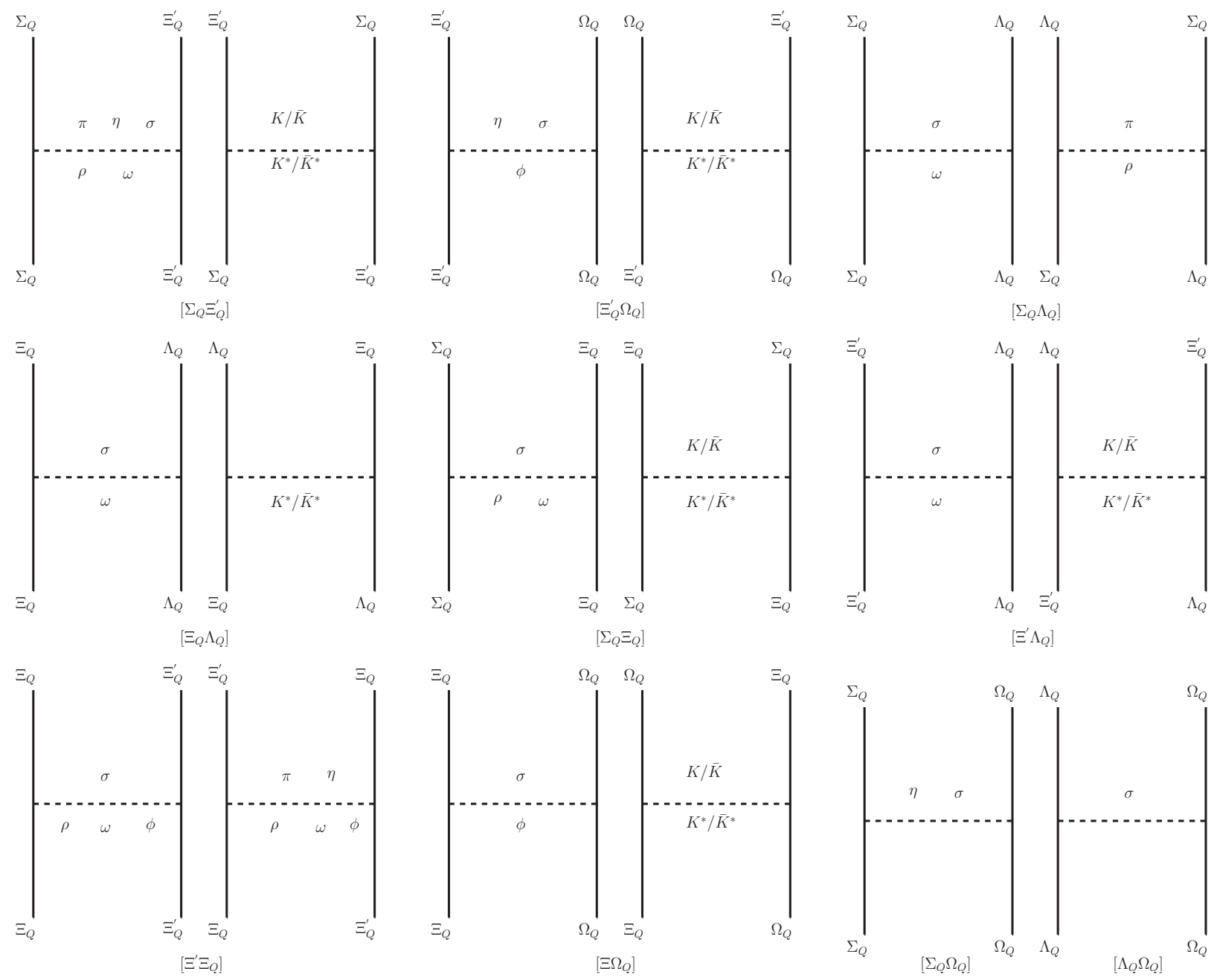

FIG. 1: The exchanged mesons for different systems. 
with $\theta_{\alpha}^{2}=-\left[m_{\alpha}^{2}-\left(m_{f}-m_{i}\right)^{2}\right]$

$$
\text { vector exchange, } \begin{aligned}
\mathcal{V}_{C}^{v}(r ; n, \beta) & =C_{n, \beta}^{v} \frac{u_{\beta}}{4 \pi}\left[g_{1 v} g_{2 v} H_{0}\left(\Lambda, m_{\beta}, r\right)+\frac{u_{\beta}^{2}}{8 m_{A} m_{B}}\left(g_{1 v} g_{2 v}+2 g_{1 v} f_{2 v}+2 g_{2 v} f_{1 v}\right) H_{0}\left(\Lambda, m_{\beta}, r\right)\right], \\
\mathcal{V}_{S S}^{v}(r ; n, \beta) & =C_{n, \beta}^{v}\left[g_{1 v} g_{2 v}+g_{1 v} f_{2 v}+g_{2 v} f_{1 v}+f_{1 v} f_{2 v}\right] \frac{1}{4 \pi} \frac{u_{\beta}^{3}}{6 m_{A} m_{B}} H_{0}\left(\Lambda, m_{\beta}, r\right) \boldsymbol{\sigma}_{A} \cdot \boldsymbol{\sigma}_{B}, \\
\mathcal{V}_{T}^{v}(r ; n, \beta) & =-C_{n, \beta}^{v}\left[g_{1 v} g_{2 v}+g_{1 v} f_{2 v}+g_{2 v} f_{1 v}+f_{1 v} f_{2 v}\right] \frac{1}{4 \pi} \frac{u_{\beta}^{3}}{12 m_{A} m_{B}} H_{3}\left(\Lambda, m_{\beta}, r\right) S_{A B}(\hat{r}), \\
\mathcal{V}_{L S}^{v}(r ; n, \beta) & =-C_{n, \beta}^{v} \frac{1}{4 \pi} \frac{u_{\beta}^{3}}{2 m_{A} m_{B}} H_{2}\left(\Lambda, m_{\beta}, r\right)\left[3 g_{1 v} g_{2 v} \boldsymbol{L} \cdot \boldsymbol{S}+4 g_{1 v} f_{2 v} \boldsymbol{L} \cdot \boldsymbol{S}_{A}+4 g_{v 2} f_{1 v} \boldsymbol{L} \cdot \boldsymbol{S}_{B}\right],
\end{aligned}
$$

$$
\begin{gathered}
\text { scalar exchange, } \mathcal{V}_{C}^{s}(r ; n=1, \sigma)=-C_{n, \sigma}^{s} u_{\sigma} \frac{g_{1 s} g_{2 s}}{4 \pi}\left[H_{0}\left(\Lambda, m_{\sigma}, r\right)-\frac{u_{\sigma}^{2}}{8 m_{A} m_{B}} H_{0}\left(\Lambda, m_{\sigma}, r\right)\right], \\
\mathcal{V}_{L S}^{s}(r ; n=1, \sigma)=-C_{n, \sigma}^{s} \frac{g_{1 s} g_{2 s}}{4 \pi} \frac{u_{\sigma}^{3}}{2 m_{A} m_{B}} H_{2}\left(\Lambda, m_{\sigma}, r\right) \boldsymbol{L} \cdot \boldsymbol{S} .
\end{gathered}
$$

$C_{n, \alpha}^{p}, C_{n, \beta}^{v}$ and $C_{n, \sigma}^{s}$ are the isospin factors. Their numerical values are given in Table $\amalg$ and the exchanged mesons are shown in Fig. 11 $\boldsymbol{L}$ is the relative orbit momentum operator between the two baryons " $A_{Q}$ " and " $B_{Q}$ ". $\boldsymbol{S}_{A}, \boldsymbol{S}_{B}$ are the spin operators of the two baryons while $\boldsymbol{S}=\boldsymbol{S}_{A}+\boldsymbol{S}_{B}$ is the total spin operator. $S_{A B}(\hat{r})=3 \sigma_{A} \cdot \boldsymbol{r} \boldsymbol{\sigma}_{B} \cdot \boldsymbol{r} / r^{2}-\boldsymbol{\sigma}_{A} \cdot \boldsymbol{\sigma}_{B}$ is the tensor operator. $g_{1 p}, g_{2 p}, \ldots$ are the coupling constants given in Eqs. (6)

$$
\begin{array}{ll}
\text { direct diagram, } & u_{\alpha}=u_{\sigma}=u_{\beta}=0, \\
\text { cross diagram, } & u_{\alpha}^{2}=m_{\alpha}^{2}-\left(m_{A}-m_{B}\right)^{2}, \quad \theta_{\alpha}^{2}=-\left[m_{\alpha}^{2}-\left(m_{A}-m_{B}\right)^{2}\right], \quad u_{\beta}^{2}=m_{\beta}^{2}-\left(m_{A}-m_{B}\right)^{2} .
\end{array}
$$

Substituting the masses of the corresponding baryons for $m_{A}$ and $m_{B}$ in Eqs. 11] [14, one obtains the effective interaction potentials. Besides Eqs. (11 12) in the one-pion-exchange (OPE) model, we need include the contributions from the other heavier exchanged mesons in the the one-boson-exchange (OBE) model. The potential within the OBE model reads

$$
\begin{aligned}
\mathcal{V}(r)= & \mathcal{V}_{C}(r)+\mathcal{V}_{S S}(r)+\mathcal{V}_{L S}(r)+\mathcal{V}_{T}(r) \\
= & {\left[\mathcal{V}_{C}^{s}(r ; n=1, \sigma)+\sum_{n, \beta} \mathcal{V}_{C}^{v}(r ; n, \beta)\right]+\left[\sum_{n, \alpha} \mathcal{V}_{S S}^{p}(r ; n, \alpha)+\sum_{n, \beta} \mathcal{V}_{S S}^{v}(r ; n, \beta)\right] } \\
& +\left[\mathcal{V}_{L S}^{s}(r ; n=1, \sigma)+\sum_{n, \beta} \mathcal{V}_{L S}^{v}(r ; n, \beta)\right]+\left[\sum_{n, \alpha} \mathcal{V}_{T}^{p}(r ; n, \alpha)+\sum_{n, \beta} \mathcal{V}_{T}^{v}(r ; n, \beta)\right] .
\end{aligned}
$$

The systems with two spin-half particles are either spin-singlet $(S=0)$ or spin-triplet $(S=1)$. For the spin-singlet, we focus on the ground state ${ }^{1} S_{0}$ while for the spin-triplet, we take both ${ }^{3} S_{1}$ and ${ }^{3} D_{1}$ into account. The wave functions can be expressed as

$$
\Psi(r)^{S}=\left.y_{S}^{S}(r)\right|^{1} S_{0}>, \quad \Psi(r)^{T}=\left.\left(\begin{array}{c}
y_{S}^{T}(r) \\
0
\end{array}\right)\right|^{3} S_{1}>+\left.\left(\begin{array}{c}
0 \\
y_{D}^{T}(r)
\end{array}\right)\right|^{3} D_{1}>,
$$

where $y_{S}^{S}(r), y_{S}^{T}(r)$ and $y_{D}^{T}$ are the radial wave functions. The operators can be written in the following matrix form:

$$
\text { spin-singlet, } \quad \sigma_{A} \cdot \sigma_{B}=-3, \quad L \cdot S=0, \quad L \cdot S_{A}=0, \quad L \cdot S_{B}=0, \quad S_{A B}(\hat{r})=0,
$$

$$
\text { spin-triplet, } \quad \sigma_{A} \cdot \sigma_{B}=\left(\begin{array}{ll}
1 & 0 \\
0 & 1
\end{array}\right), \quad S_{A B}(\hat{r})=\left(\begin{array}{cc}
0 & \sqrt{8} \\
\sqrt{8} & -2
\end{array}\right), \quad \boldsymbol{L} \cdot \boldsymbol{S}=\left(\begin{array}{cc}
0 & 0 \\
0 & -3
\end{array}\right), \quad \boldsymbol{L} \cdot \boldsymbol{S}_{A}=\left(\begin{array}{cc}
0 & 0 \\
0 & -\frac{3}{2}
\end{array}\right), \boldsymbol{L} \cdot \boldsymbol{S}_{B}=\left(\begin{array}{cc}
0 & 0 \\
0 & -\frac{3}{2}
\end{array}\right) \text {. }
$$

\section{THE COUPLED CHANNEL ANALYSIS OF THE $\Lambda_{Q} \Lambda_{Q}$ SYSTEM}

The $\Lambda_{Q} \Lambda_{Q}\left[0\left(0^{+}\right)\right]$system is very interesting, which can be viewed as the heavy analogue of the $\mathrm{H}$ dibaryon. The heavy quark mass $m_{b, c}$, the S-D wave mixing and the coupled channel effect in the flavor space all may play an important role in the 
TABLE III: The isospin factors. The superscript in the first column is the isospin of the system. The values outside the square brackets are for the direct diagram while the ones inside the square brackets are for the crossed diagram.

\begin{tabular}{ccccccccc}
\hline States & $C_{n, \pi}^{p}$ & $C_{n, \eta}^{p}$ & $C_{n, K}^{p}$ & $C_{n, \rho}^{v}$ & $C_{n, \omega}^{v}$ & $C_{n, \phi}^{v}$ & $C_{n, K^{*}}^{p}$ & $C_{n, \sigma}^{s}$ \\
\hline$\left[\Sigma_{Q} \Xi_{Q}^{\prime}\right]^{I=1 / 2}$ & $-1 / 2$ & $-1 / 12$ & {$[-1 / 4]$} & $-1 / 2$ & $1 / 4$ & & {$[-1 / 4]$} & 1 \\
{$\left[\Sigma_{Q} \Xi_{Q}^{\prime}\right]^{I=3 / 2}$} & $1 / 4$ & $-1 / 12$ & {$[1 / 2]$} & $1 / 4$ & $1 / 4$ & & {$[1 / 2]$} & 1 \\
{$\left[\Xi_{Q}^{\prime} \Omega_{Q}\right]^{I=1 / 2}$} & & $1 / 6$ & {$[1 / 2]$} & & & $1 / 2$ & {$[1 / 2]$} & 1 \\
{$\left[\Sigma_{Q} \Omega_{Q}\right]^{I=1 / 2}$} & & $-1 / 3$ & & & & & 1 \\
{$\left[\Xi_{Q} \Lambda_{Q}\right]^{I=1 / 2}$} & & & & & 1 & & {$[1]$} & 4 \\
{$\left[\Sigma_{Q} \Lambda_{Q}\right]^{I=1}$} & {$[1]$} & & & {$[1]$} & 1 & & 2 \\
{$\left[\Sigma_{Q} \Xi_{Q}\right]^{I=1 / 2}$} & & & {$[-1 / 2]$} & -1 & $1 / 2$ & & {$[-1 / 2]$} & 2 \\
{$\left[\Sigma_{Q} \Xi_{Q}\right]^{I=3 / 2}$} & & & {$[1]$} & $1 / 2$ & $1 / 2$ & & {$[1]$} & 2 \\
{$\left[\Xi_{Q}^{\prime} \Lambda_{Q}\right]^{I=1 / 2}$} & & & {$[1 / 2]$} & & $1 / 2$ & & {$[1 / 2]$} & 2 \\
{$\left[\Xi_{Q} \Xi_{Q}^{\prime}\right]^{I=0}$} & {$[3 / 4]$} & {$[-3 / 4]$} & & $-3 / 4[3 / 4]$ & $1 / 4[-1 / 4]$ & $1 / 2[-1 / 2]$ & & 2 \\
{$\left[\Xi_{Q} \Xi_{Q}^{\prime}\right]^{I=1}$} & {$[1 / 4]$} & {$[3 / 4]$} & & $1 / 4[1 / 4]$ & $1 / 4[1 / 4]$ & $1 / 2[1 / 2]$ & & 2 \\
{$\left[\Xi_{Q} \Omega_{Q}\right]^{I=1 / 2}$} & & & {$[1]$} & & & 1 & {$[1]$} & 2 \\
{$\left[\Lambda_{Q} \Omega_{Q}\right]^{I=0}$} & & & & & & & \\
\hline
\end{tabular}

formation of the possible loosely bound states. Investigation and comparison of the $\Lambda_{b} \Lambda_{b}, \Lambda_{c} \Lambda_{c}$, and $\Lambda \Lambda$ systems may reveal which underlying mechanism is dominant.

In the present work we shall perform an extensive analysis of $\Lambda_{Q} \Lambda_{Q}$ with quantum numbers $I\left(J^{P}\right)=0\left(0^{+}\right), 0\left(0^{-}\right)$and $0\left(1^{-}\right)$. We list the flavor channels which we take into account in Table IV] Besides the lagrangians given in Eqs. (3] [5), we also need the following effective Lagrangians:

$$
\begin{aligned}
\text { pseudoscalar exchange, } \mathcal{L}_{p}= & {\left[-\frac{g_{3}}{\sqrt{2} f_{\pi}} \operatorname{Tr}\left(\bar{B}_{6 \mu}^{*} \partial^{\mu} M B_{6}\right)+\text { h.c. }\right]+\left[-\frac{g_{4}}{\sqrt{2} f_{\pi}} \operatorname{Tr}\left(\bar{B}_{6 \mu}^{*} \partial^{\mu} M B_{\overline{3}}\right)+\text { h.c. }\right] } \\
& -\frac{g_{5}}{\sqrt{2} f_{\pi}} \operatorname{Tr}\left(\bar{B}_{6 \mu}^{*} \gamma_{v} \gamma_{5} \partial^{v} M B_{6}^{* \mu}\right) \\
\text { scalar exchange, } \quad \mathcal{L}_{s}= & l_{s} \operatorname{Tr}\left(\bar{B}_{6 \mu}^{*} \sigma B_{6}^{* \mu}\right), \\
\text { vector exchange, } \quad \mathcal{L}_{v}= & \frac{\beta_{s} g_{v}}{\sqrt{2}} \operatorname{Tr}\left(\bar{B}_{6 \mu}^{*} \gamma_{v} V^{v} B_{6}^{* \mu}\right)+\frac{i \lambda_{s} g_{v}}{\sqrt{2}} \operatorname{Tr}\left[\bar{B}_{6 \mu}^{*}\left(\partial^{\mu} V^{v}-\partial^{v} V^{\mu}\right) B_{6 v}^{*}\right] \\
& +\left\{-\frac{i \lambda_{s} g_{v}}{\sqrt{6}} \operatorname{Tr}\left[\bar{B}_{6 \mu}^{*}\left(\partial^{\mu} V^{v}-\partial^{v} V^{\mu}\right) \gamma_{v} \gamma_{5} B_{6}\right]+\text { h.c. }\right\} \\
& +\left\{-i \sqrt{2} \lambda_{I} g_{v} \operatorname{Tr}\left[B_{6 \mu}^{*}\left(\partial^{\mu} V^{v}-\partial^{v} V^{\mu}\right) \gamma_{v} \gamma_{5} B_{\overline{3}}\right]+\text { h.c. }\right\} .
\end{aligned}
$$

The coupling constants are $g_{4}=0.999, g_{3}=\sqrt{6} g_{4}, g_{5}=-\sqrt{2} g_{4}, l_{s}=6.2, f_{\pi}=92.3 \mathrm{MeV},\left(\beta_{s} g_{v}\right)=12,\left(\lambda_{s} g_{v}\right)=19.2 \mathrm{GeV}^{-1}$ and $\left(\lambda_{I} g_{v}\right)=-\left(\lambda_{s} g_{v}\right) / \sqrt{8}[20]$. Besides the potentials in Eqs. 111][14], we also need the following potentials

$$
\begin{aligned}
\mathcal{V}^{p}(r) & =C^{p}(i, j) \frac{u^{3}}{24 \pi}\left[H_{3}\left(\Lambda, m_{e x}, r\right) \Delta_{\text {ten }}+H_{0}\left(\Lambda, m_{e x}, r\right) \Delta_{S S}\right], \\
\mathcal{V}^{v}(r) & =C_{1}^{v}(i, j) \frac{u}{4 \pi} H_{0}\left(\Lambda, m_{e x}, r\right)+C_{2}^{v}(i, j) \frac{u^{3}}{12 \pi}\left[-H_{3}\left(\Lambda, m_{e x}, r\right) \Delta_{t e n}+2 H_{0}\left(\Lambda, m_{e x}, r\right) \Delta_{S S}\right], \\
\mathcal{V}^{s}(r) & =C^{s}(i, j) \frac{m_{\sigma}}{4 \pi} H_{0}\left(\Lambda, m_{\sigma}, r\right) .
\end{aligned}
$$

where $\Delta_{t e n}$ and $\Delta_{S S}$ denote the tensor and spin-spin operators respectively. They are channel-dependent. Their specific expressions are given in Table $\nabla$

For the baryon masses, we use $m_{\Sigma_{c}^{*}}=2518.0 \mathrm{MeV}$ and $m_{\Sigma_{b}^{*}}=5832.5 \mathrm{MeV}$ [28]. Due to the conservation of the energy and 
momentum, we keep the non-vanishing zeroth component of the exchanged four momentum $Q_{0}$ and define $u$ as the following

$$
\begin{aligned}
& \Sigma_{Q} \Sigma_{Q}^{*} \leftrightarrow \Sigma_{Q}^{*} \Sigma_{Q}, \quad u^{2}=m_{e x}^{2}-\left(m_{\Sigma_{Q}^{*}}-m_{\Sigma_{Q}}\right)^{2}, \quad \Lambda_{Q} \Lambda_{Q} \leftrightarrow \Sigma_{Q} \Sigma_{Q}^{*}, \quad u^{2}=m_{e x}^{2}-\left(\frac{m_{\Sigma_{Q}^{*}}^{2}-m_{\Sigma_{Q}}^{2}}{4 m_{\Lambda_{Q}}}\right)^{2}, \\
& \Sigma_{Q} \Sigma_{Q} \leftrightarrow \Sigma_{Q} \Sigma_{Q}^{*}, \quad u^{2}=m_{e x}^{2}-\left(\frac{m_{\Sigma_{Q}^{*}}^{2}-m_{\Sigma_{Q}}^{2}}{4 m_{\Sigma_{Q}}}\right)^{2}, \quad \Sigma_{Q}^{*} \Sigma_{Q}^{*} \leftrightarrow \Sigma_{Q} \Sigma_{Q}^{*}, \quad u^{2}=m_{e x}^{2}-\left(\frac{m_{\Sigma_{Q}^{*}}^{2}-m_{\Sigma_{Q}}^{2}}{4 m_{\Sigma_{Q}}^{*}}\right)^{2}, \text { Other channels, } u^{2}=m_{e x}^{2} .
\end{aligned}
$$

\begin{tabular}{|c|c|c|c|c|c|c|c|}
\hline$I\left(J^{P}\right)=0\left(0^{+}\right)$ & $\Lambda_{Q} \Lambda_{Q}\left({ }^{1} S_{0}\right)$ & $\Sigma_{Q} \Sigma_{Q}\left({ }^{1} S_{0}\right)$ & $\Sigma_{Q}^{*} \Sigma_{Q}^{*}\left({ }^{1} S_{0}\right)$ & $\Sigma_{Q} \Sigma_{Q}^{*}\left({ }^{5} D_{0}\right)$ & $\Sigma_{Q}^{*} \Sigma_{Q}^{*}\left({ }^{5} D_{0}\right)$ & & \\
\hline$I\left(J^{P}\right)=0\left(1^{-}\right)$ & $\Lambda_{Q} \Lambda_{Q}\left({ }^{3} P_{1}\right)$ & $\Sigma_{Q} \Sigma_{Q}\left({ }^{3} P_{1}\right)$ & $\Sigma_{Q}^{*} \Sigma_{Q}^{*}\left({ }^{3} P_{1}\right)$ & $\Sigma_{Q}^{*} \Sigma_{Q}^{*}\left({ }^{7} F_{1}\right)$ & $\Sigma_{Q} \Sigma_{Q}^{*}\left({ }^{3} P_{1}\right)$ & $\Sigma_{Q} \Sigma_{Q}^{*}\left({ }^{5} P_{1}\right)$ & $\Sigma_{Q} \Sigma_{Q}^{*}\left({ }^{5} F_{1}\right)$ \\
\hline
\end{tabular}

TABLE IV: The flavor channels for the $\Lambda_{Q} \Lambda_{Q}$ system, where $Q=b$ or $c$.

\section{NUMERICAL RESULTS FOR THE $A_{Q} B_{Q}$ SYSTEMS}

In our numerical analysis, we apply the Fortran program FESSDE [29] to solve the multichannel Schrödinger equation. Solving the Schödinger equations with the potentials derived in the previous sections, we obtain the numerical results including the binding energy (B.E.), the root-mean-square radius $\left(r_{r m s}\right)$ and the probabilities of the individual channels. We also plot the dependence of the binding energy on the cutoff parameter in the Appendix.

The hadronic molecule is a loosely bound state. Its constituents are expected to be well separated. One expects that the size of the molecules should be much larger than that of the conventional $q \bar{q}$ and $q q q$ hadrons. Recall that the size of the deuteron is about $1.96 \mathrm{fm}$ [22]. Törnqvist argued that the size of the meson-meson molecule is even up to $3 \mathrm{fm}$ [21]. We expect the size of the hadronic molecules composed of two heavy baryons should be comparable to the size of the deuteron. The size of the molecular system may tell us whether the present framework and numerical results are self-consistent or not. To be more specific, the size of the molecular states composed of two charmed (or bottomed) baryons is expected to be larger than that of $J / \psi$ (or Upsilon).

Generally the value of the cutoff parameter is determined through fit to experimental data. In our case, there is almost no information on the heavy baryon-baryon interaction through which we can extract the cutoff parameter. Fortunately, the oneboson-exchange model is very successful in explaining the deuteron with the cutoff parameter $0.80 \mathrm{GeV}<\mathrm{cutoff}<1.5 \mathrm{GeV}$, which provides us a good benchmark.

The present OBE model is rather crude. For example, we adopt the same cutoff parameter for all the meson exchange. We plot the interaction potentials of the deuteron with the OBE model in Fig. 2] We present the numerical results in Table VI

TABLE V: The specific expressions of operators $\Delta_{t e n}$ and $\Delta_{S S}$ for the individual channels. $S_{t}$ and $\frac{3}{2} \sigma_{r s} \equiv-\frac{3}{2} S_{t \mu} \sigma S^{t \mu}$ are the transition matrix and the spin operator of the spin- $\frac{3}{2}$ baryons, respectively. One can refer to Ref. [20] for their definitions.

\begin{tabular}{ccc}
\hline Channels & $\Delta_{t e n}$ & $\Delta_{S S}$ \\
\hline$\Lambda_{Q} \Lambda_{Q} \leftrightarrow \Sigma_{Q}^{*} \Sigma_{Q}^{*}$ & $3 \mathbf{S}_{t 1}^{\dagger} \cdot \hat{\mathbf{r}} \mathbf{S}_{t 2}^{\dagger} \cdot \hat{\mathbf{r}}-\mathbf{S}_{t 1}^{\dagger} \cdot \mathbf{S}_{t 2}^{\dagger}$ & $\mathbf{S}_{t 1}^{\dagger} \cdot \mathbf{S}_{t 2}^{\dagger}$ \\
$\Sigma_{Q} \Sigma_{Q} \leftrightarrow \Sigma_{Q}^{*} \Sigma_{Q}^{*}$ & & \\
$\Lambda_{Q} \Lambda_{Q} \leftrightarrow \Sigma_{Q} \Sigma_{Q}^{*}$ & $3 \sigma_{1} \cdot \hat{\mathbf{r}} \mathbf{S}_{t 2}^{\dagger} \cdot \hat{\mathbf{r}}-\sigma_{1} \cdot \mathbf{S}_{t 2}^{\dagger}$ & $\sigma_{1} \cdot \mathbf{S}_{t 2}^{\dagger}$ \\
$\Sigma_{Q} \Sigma_{Q} \leftrightarrow \Sigma_{Q} \Sigma_{Q}^{*}$ & $3 \sigma_{r s 1} \cdot \hat{\mathbf{r}} \sigma_{r s 2} \cdot \hat{\mathbf{r}}-\sigma_{r s 1} \cdot \sigma_{r s 2}$ & \\
$\Sigma_{Q}^{*} \Sigma_{Q}^{*} \leftrightarrow \Sigma_{Q}^{*} \Sigma_{Q}^{*}$ & $3 \sigma_{1} \cdot \hat{\mathbf{r}} \sigma_{r s 2} \cdot \hat{\mathbf{r}}-\sigma_{1} \cdot \sigma_{r s 2}$ & $\sigma_{r s 1} \cdot \sigma_{r s 2}$ \\
$\Sigma_{Q} \Sigma_{Q}^{*} \leftrightarrow \Sigma_{Q} \Sigma_{Q}^{*}$ & $3 \mathbf{S}_{t 1}^{\dagger} \cdot \hat{\mathbf{r}} \mathbf{S}_{t 2} \cdot \hat{\mathbf{r}}-\mathbf{S}_{t 1}^{\dagger} \cdot \mathbf{S}_{t 2}$ & $\sigma_{1} \cdot \sigma_{r s 2}$ \\
$\Sigma_{Q} \Sigma_{Q}^{*} \leftrightarrow \Sigma_{Q}^{*} \Sigma_{Q}$ & & $\mathbf{S}_{t 1}^{\dagger} \cdot \mathbf{S}_{t 2}$ \\
\hline
\end{tabular}



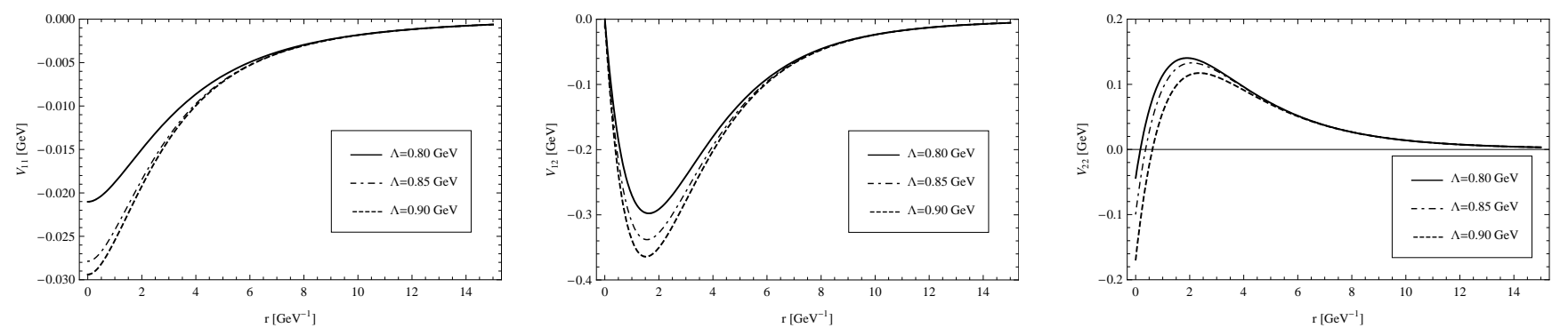

FIG. 2: The interaction potentials of the deuteron within the OBE model. $V_{11}, V_{12}$ and $V_{22}$ are for the transitions ${ }^{3} S_{1} \leftrightarrow{ }^{3} S_{1},{ }^{3} S_{1} \leftrightarrow{ }^{3} D_{1}$ and ${ }^{3} D_{1} \leftrightarrow{ }^{3} D_{1}$, respectively.

TABLE VI: The numerical result of the deuteron with the OBE potential. " $\Lambda$ " is the cutoff parameter. "B.E." means the binding energy. " $P_{S}$ " and " $P_{D}$ " are the probabilities of the $\mathrm{S}$ wave and $\mathrm{D}$ wave respectively.

\begin{tabular}{cccccccccc}
\hline \multicolumn{4}{c}{ without contact term } \\
$\Lambda(\mathrm{GeV})$ & B.E. $(\mathrm{MeV})$ & $r_{r m s}(\mathrm{fm})$ & $P_{S}(\%)$ & $P_{D}(\%)$ & $\Lambda(\mathrm{GeV})$ & B.E. $(\mathrm{MeV})$ & $r_{r m s}(\mathrm{fm})$ & $P_{S}(\%)$ & $P_{D}(\%)$ \\
\hline 0.80 & 9.31 & 2.14 & 93.19 & 6.81 & 0.80 & 1.87 & 4.06 & 95.00 & 5.00 \\
0.85 & 18.77 & 1.65 & 92.40 & 7.60 & 0.85 & 2.58 & 3.59 & 94.14 & 5.86 \\
0.90 & 29.45 & 1.39 & 92.14 & 7.86 & 0.90 & 2.88 & 3.37 & 93.82 & 6.18 \\
\hline
\end{tabular}

In order to study the effect of the contact interaction, we compare the results (1) when the $\delta(r)$ function is omitted and (2) when the $\delta(r)$ function is explicitly kept. Without the contact interaction piece, the binding energy of the deuteron is 9.31 $\mathrm{MeV}$ and the root-mean-square radius is $2.14 \mathrm{fm}$ when the cutoff is $0.8 \mathrm{GeV}$. If we include the contact interaction, the binding energy decreases to $1.87 \mathrm{MeV}$ and the root-mean-square radius increases to $4.06 \mathrm{fm}$. In other words, both approaches roughly reproduce the qualitative feature of the loosely bound deuteron. In the following, we present the numerical results without the contact interaction. For comparison, we collect the results with the contact interaction in Appendix VIIB

However, if we shut down the ${ }^{3} D_{1}$ channel, we can not find the binding solutions, which means that the S-D mixing effect is very important in the formation of the loosely deuteron bound state although the probability of the $\mathrm{D}$ wave is as small as $\sim 6 \%$. One can refer to Fig. 3 for the variations of the binding energy and the root-mean-square radius of the deuteron with the cutoff parameter.

It's interesting to investigate whether the long-range pion-exchange interaction plays a dominant role in forming the hadronic molecules. Therefore, in the first part we give the numerical results with the one-pion-exchange (OPE) potential for the systems where the pion exchange is allowed. In the second part we take into account the scalar and vector boson exchanges, which account for the medium- and short-range interactions, as well as the $\pi$ exchange.
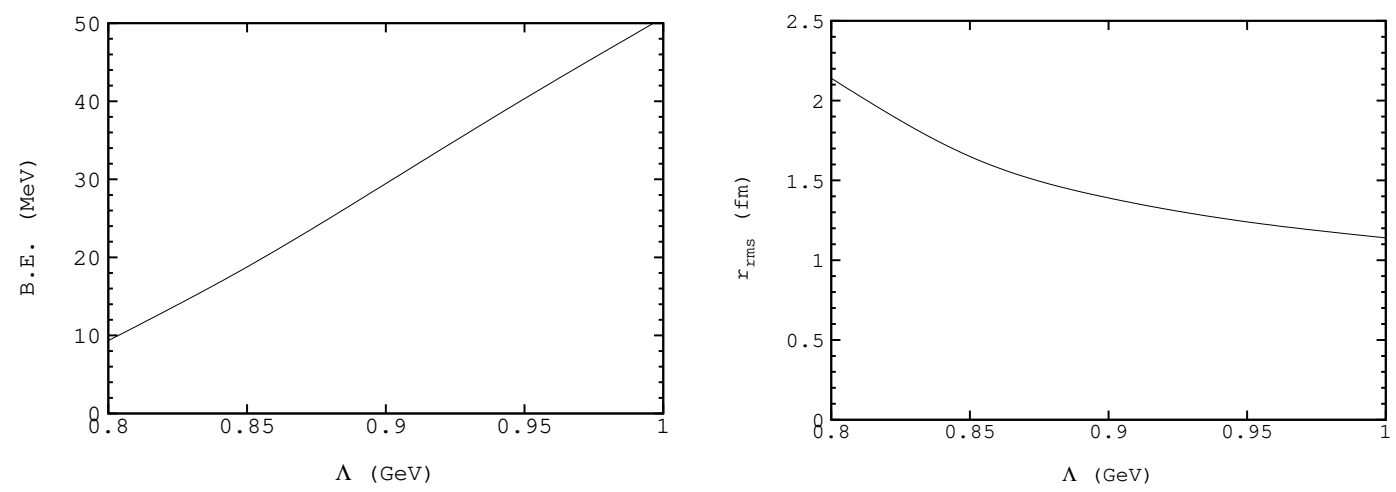

FIG. 3: The variations of the binding energy and the root-mean-square radius of the deuteron with the cutoff parameter. 
TABLE VII: The numerical results for the spin-triplet system $(S=1)$ with the OPE potential. " $X$ " indicates no binding solutions. $\Lambda$ is the cutoff parameter. B.E. is the binding energy while $r_{r m s}$ is the root-mean-square radius. $P_{S}$ and $P_{D}$ indicate the the probabilities of the $\mathrm{S}$ wave and the D-wave, respectively. $Q=c$ or $b$ denotes the charmed or the bottomed systems.

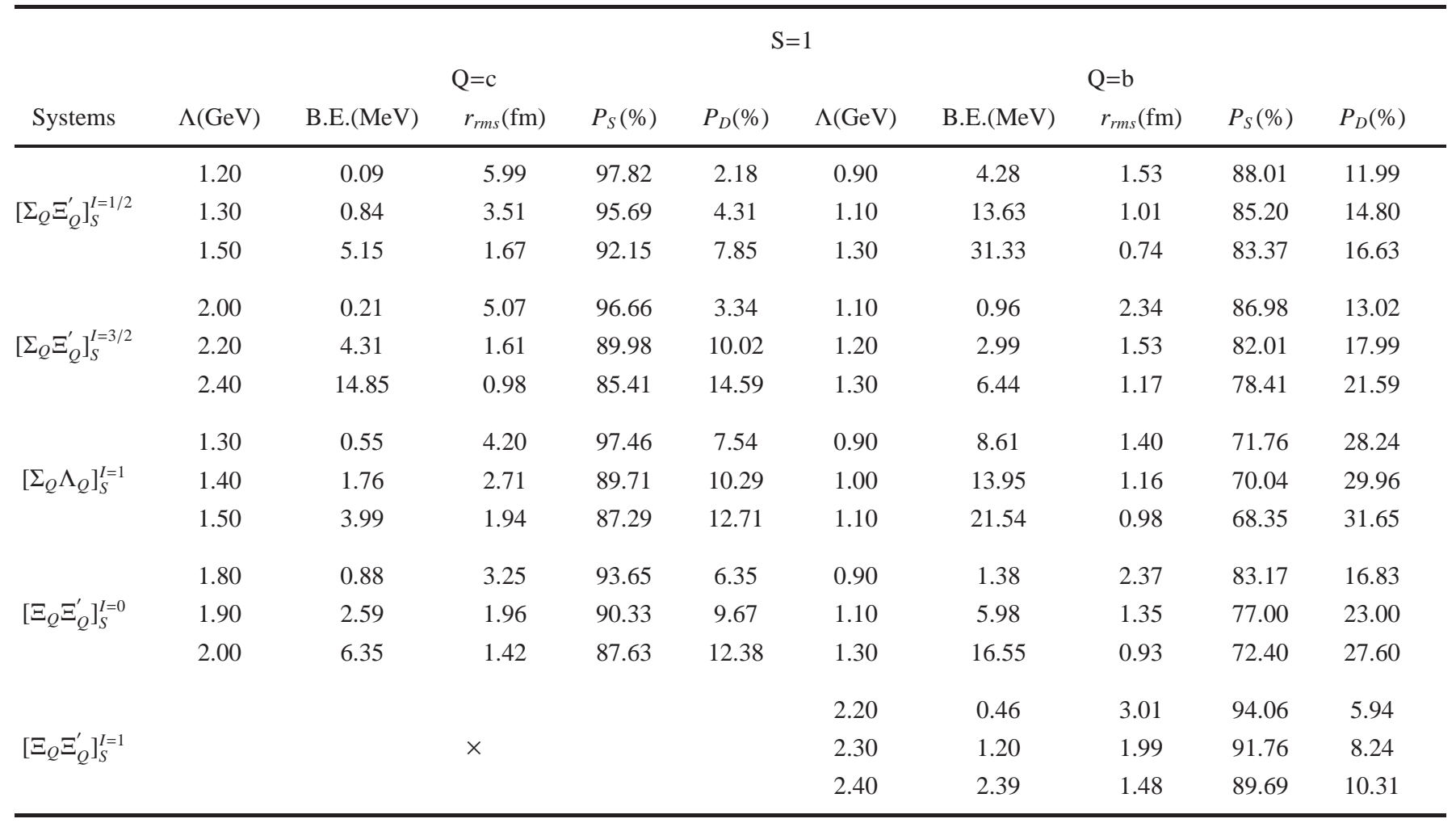

\section{A. The Results of The " $A_{Q} B_{Q}$ " Systems with The OPE Potential}

From Fig. 11 and Table III, we can see that there exists the one-pion-exchange force for five systems: $\left[\Sigma_{Q} \Xi_{Q}^{\prime}\right]_{S}^{(I=1 / 2,3 / 2)}$, $\left[\Sigma_{Q} \Lambda_{Q}\right]_{S}^{I=1}$ and $\left[\Xi_{Q} \Xi_{Q}^{\prime}\right]_{S}^{(I=0,1)}$. For $\left[\Sigma_{Q} \Xi_{Q}^{\prime}\right]_{S}^{I=1 / 2}$ and $\left[\Sigma_{Q} \Xi_{Q}^{\prime}\right]_{S}^{3 / 2}$, the pion exchange exists in the direct channel while the pion exchange occurs in the cross channel for the other three systems.

There are no binding solutions for the five spin-singlet $(S=0)$ systems. For the spin-triplet $(S=1)$ case, we list the numerical results in Table VII and plot the dependence of the binding energy on the cutoff parameter in Fig. 5. We obtain binding solutions for all the states except for $\left[\Xi_{c} \Xi_{c}^{\prime}\right]_{S=1}^{I=1}$, see Table VII] In the charmed sector, loosely bound states $\left[\Sigma_{c} \Xi_{c}^{\prime}\right]_{S=1}^{I=1 / 2}$ and $\left[\Sigma_{c} \Lambda_{c}\right]_{S=1}^{I=1}$ have small binding energy around a few $\mathrm{MeV}$ for a reasonable cutoff parameter about $1.20 \sim 1.50 \mathrm{GeV}$. They are good candidates of molecules. For these two states, the D wave contribution is less than $13 \%$. For the states $\left[\Sigma_{c} \Xi_{c}^{\prime}\right]_{S=1}^{I=3 / 2}$ and $\left[\Xi_{c} \Xi_{c}^{\prime}\right]_{S=1}^{I=0}$, the binding solutions exist with the cutoff parameter around $2.0 \mathrm{GeV}$ and $1.80 \mathrm{GeV}$, respectively. And, the D wave probabilities for the two states are less than $15 \%$.

The bottomed case is similar to the charmed case except that the binding energy of the bottomed bound states is deeper. This is mainly because that the larger mass of the bottomed baryon reduces the kinetic energy. For the states $\left[\Sigma_{b} \Xi_{b}^{\prime}\right]_{S=1}^{(I=1 / 2,3 / 2)},\left[\Sigma_{b} \Lambda_{b}\right]_{S=1}^{I=1}$ and $\left[\Xi_{b} \Xi_{b}^{\prime}\right]_{S=1}^{I=0}$, we obtain loosely bound states with binding energy less than $30 \mathrm{MeV}$ and the root-mean-square radius larger than $0.7 \mathrm{fm}$, when the cutoff parameter is about $0.9 \sim 1.30 \mathrm{GeV}$. The $\mathrm{D}$ wave contribution is larger for the bottomed systems than for the charmed systems, see Table VII. In [30], the authors performed a study of the $\Sigma_{Q} \Lambda_{Q}$ system at the quark level and obtained larger binding energy. The $\left[\Xi_{b} \Xi_{b}^{\prime}\right]_{S=1}^{I=1}$ bound state appears when the cutoff parameter is $2.20 \mathrm{GeV}$. When $2.20 \mathrm{GeV}<\mathrm{cutoff}<2.40$ $\mathrm{GeV}$, the binding energy is $0.46 \sim 2.39 \mathrm{MeV}$.

By comparing the numerical results of the two pairs of isospin multiplets, $\left[\Sigma_{Q} \Xi_{Q}^{\prime}\right]_{S}^{I=1 / 2}$ and $\left[\Sigma_{Q} \Xi_{Q}^{\prime}\right]_{S}^{I=3 / 2}$ and $\left[\Xi_{Q} \Xi_{Q}^{\prime}\right]_{S}^{I=0}$ and $\left[\Xi_{Q} \Xi_{Q}^{\prime}\right]_{S}^{I=1}$, we can see that the results are different for different isospin multiplets of the same flavor system since the potentials are isospin-dependent. Comparing the results of the charmed systems with those of the bottomed systems, it is obvious that the large heavy quark mass is salient in the formation of the molecular states. 


\section{B. The Results of The " $A_{Q} B_{Q}$ " Systems with The OBE Potential}

In the previous subsection, we give the numerical results with the $\pi$ exchange potential which accounts for the long-range interaction. Actually, only five systems out of the thirteen ones allow the $\pi$ exchange. We find that the $\pi$ exchange is not strong enough to form bound states for all the five spin-singlet systems. In order to make the individual role of the exchanged boson clear, we also give the numerical results within the one-boson-exchange model (OBE) in Tables VIII IX] and plot the dependence of binding energy on the cutoff parameter in Figs. 60.

In the spin-singlet case, we still find no binding solutions for the state $\left[\Sigma_{Q} \Xi_{Q}^{\prime}\right]_{S=0}^{I=1 / 2}(\mathrm{Q}=\mathrm{b}, \mathrm{c})$ even if we add the contributions of the heavier vector and scalar meson exchange. Therefore, our results disfavor the existence of the molecules $\left[\Sigma_{Q} \Xi_{Q}^{\prime}\right]_{S=0}^{I=1 / 2}$ $(\mathrm{Q}=\mathrm{b}, \mathrm{c})$. However, for the other isospin multiplet of this state with $I=\frac{3}{2}$, we find binding solutions for both the charmed and bottomed cases. A bound state of $\left[\Sigma_{c} \Xi_{c}^{\prime}\right]_{S=1}^{I=3 / 2}$ appears with binding energy about $3.54 \sim 67.46 \mathrm{MeV}$ when the cutoff parameter is around $0.8 \sim 1.0 \mathrm{GeV}$. The binding energy of the corresponding bottomed state $\left[\Sigma_{b} \Xi_{b}^{\prime}\right]_{S=0}^{I=3 / 2}$ is about $156.78 \mathrm{MeV}$ when the cutoff parameter is $1.0 \mathrm{GeV}$. Such a large binding energy seems too deep for a loosely bound molecular state. For the $\left[\Sigma_{Q} \Lambda_{Q}\right]_{S=0}^{I=1}$ system, the binding energy of the state $\left[\Sigma_{c} \Lambda_{c}\right]_{S=0}^{I=1}$ is $0.28 \sim 47.34 \mathrm{MeV}$ with the cutoff parameter $0.90 \sim 1.10 \mathrm{MeV}$ while the binding energy of $\left[\Sigma_{b} \Lambda_{b}\right]_{S=0}^{I=1}$ is $0.34 \sim 62.13 \mathrm{MeV}$ with a cutoff parameter $0.80 \sim 1.00 \mathrm{GeV}$. We obtain bound states for both the charmed and the bottomed cases for $\left[\Xi_{Q} \Xi_{Q}^{\prime}\right]$ with $I=0$ and $I=1$.

For the other systems without the $\pi$ exchange, we also find binding solutions. The most interesting one may be $\left[\Xi_{Q} \Lambda_{Q}\right]_{S=0}^{I=1 / 2}$, which allows the $\sigma$ and $\omega$ exchanges in the direct channel and $K^{*} / \bar{K}^{*}$ exchange in the cross channel. For the charmed case, a very loosely bound states with binding energy $1.91 \sim 3.03 \mathrm{MeV}$ appears when the cutoff parameter is $1.10 \sim 1.50 \mathrm{GeV}$. For the bottomed case, a bound state emerges with binding energy $10.33 \sim 28.65 \mathrm{MeV}$ when the cutoff parameter is between $0.90 \mathrm{GeV}$ and $1.50 \mathrm{GeV}$. They are very good molecule candidates. For the states $\left[\Sigma_{c} \Xi_{c}\right]_{S=0}^{I=1 / 2}$ and $\left[\Sigma_{c} \Omega_{c}\right]_{S=0}^{I=1}$, we also obtain small binding energies and large root-mean-square radii with reasonable cutoff parameter $1.0 \sim 1.50 \mathrm{GeV}$ as shown in Table VIII. Our results are in favor of the existences of these molecular states. The binding energy of $\left[\Xi_{b}^{\prime} \Omega_{b}\right]_{S=0}^{I=1 / 2}$ is $80.49 \sim 107.01 \mathrm{MeV}$ with cutoff parameter $0.90 \sim 1.00 \mathrm{GeV}$. Again, such a large binding energy seems too deep for a loosely bound molecular state.

In the spin-triplet sector, it is interesting to compare with the deuteron case. We plot the interaction potential of the $\Sigma_{c} \Xi_{c}^{\prime}\left[I\left(J^{P}\right)=\frac{1}{2}\left(1^{+}\right)\right]$system in Fig. 4 From Fig. 2 and Fig. 4 it is clear that the potentials of the two systems are similar. Their binding solutions are also similar except that the $\Sigma_{c} \Xi_{c}^{\prime}$ system has even shallower binding energy and smaller D wave probability, as can be seen from Tables VI and IX]

The bound state of $\left[\Sigma_{c} \Xi_{c}^{\prime}\right]_{S=1}^{I=3 / 2}$ disappears if we take the heavier scalar and vector meson exchanges into account. There is still no binding solution for the state $\left[\Xi_{c} \Xi_{c}^{\prime}\right]_{S=1}^{I=1}$ when we consider all the contributions of the exchanged mesons. The binding energy of the state $\left[\Sigma_{Q} \Lambda_{Q}\right]_{S=1}^{I=1}$ becomes shallower in the OBE model. For the other systems with the $\pi$ exchange, the numerical results within the OBE model are similar to those within the OPE model except that the binding energy becomes deeper as shown in Table IX

From Table IX one can see that there is no S-D mixing for the two states $\left[\Xi_{Q} \Lambda_{Q}\right]_{S=1}^{I=1 / 2}$ and $\left[\Lambda_{Q} \Omega_{Q}\right]_{S=1}^{I=0}$. Actually, for these two systems the results are the same for both the spin-singlet and spin-triplet cases because the potential is the same. For the states $\left[\Xi_{c}^{\prime} \Omega_{c}\right]_{S=1}^{I=1 / 2}$ and $\left[\Sigma_{c} \Xi_{c}\right]_{S=1}^{I=3 / 2}$, there are no binding solutions. However, a very loosely bound state $\left[\Xi_{c}^{\prime} \Lambda_{c}\right]_{S=1}^{I=1 / 2}$ exists with binding energy $0.17 \sim 0.69 \mathrm{MeV}$ when the cutoff parameter is around $1.00 \sim 1.40 \mathrm{GeV}$. The binding energy of its bottomed counterpart $\left[\Xi_{b}^{\prime} \Lambda_{b}\right]_{S=1}^{I=1 / 2}$ is $17.64 \sim 23.91 \mathrm{MeV}$ with cutoff parameter around $1.0 \sim 1.40 \mathrm{GeV}$. We also obtain a loosely bound state $\left[\Xi_{c} \Omega_{c}\right]_{S=1}^{I=1 / 2}$ with binding energy $2.63 \sim 4.51 \mathrm{MeV}$ when the cutoff parameter is between $1.00 \mathrm{GeV}$ and $1.20 \mathrm{GeV}$. Once these three molecule states are produced, they should be very stable because their constituents $\Lambda_{Q}$, $\Xi_{Q}^{\prime}$, $\Xi_{Q}$ and $\Omega_{Q}$ decay via weak interaction. Comparing the results of the OBE model with those of the OPE model, one can see that the contribution of the $\mathrm{D}$ wave decreases if we take into account the scalar and vector meson exchange, which implies that the S-D mixing mainly comes from the $\pi$ exchange.

\section{NUMERICAL RESULTS FOR THE $\Lambda_{Q} \Lambda_{Q}$ SYSTEM}

We investigated the $\Lambda_{Q} \Lambda_{Q}\left[I\left(J^{P}\right)=0\left(0^{+}\right)\right]$system with the $\sigma$ and $\omega$ exchange potential, but without the coupled-channel effect in the flavor space in Ref. [19]. We find no binding solutions. Later, the authors in Ref. [20] considered the coupled-channel effect and studied this state using the pion-exchange potential. They found a bound state solution. It is intriguing to study the variation of the bound state solution with the heavy quark mass, the S-D mixing effect, the long-range OPE force and medium/short-range interaction respectively. In the first subsection, we shall present the numerical results for the OPE model with the coupled-channel effect. In the second subsection, we will add the scalar and vector meson exchange force which also contributes to the transition in the flavor space. 
TABLE VIII: The numerical results for the spin-singlet $(S=0)$ case with the OBE potential. $\times$ means no binding solutions exist.

\begin{tabular}{|c|c|c|c|c|c|c|}
\hline \multirow[b]{3}{*}{ Systems } & \multicolumn{6}{|c|}{$\mathrm{S}=0$} \\
\hline & \multicolumn{3}{|c|}{$\mathrm{Q}=\mathrm{c}$} & \multicolumn{3}{|c|}{$\mathrm{Q}=\mathrm{b}$} \\
\hline & $\Lambda(\mathrm{GeV})$ & B.E.(MeV) & $r_{r m s}(\mathrm{fm})$ & $\Lambda(\mathrm{GeV})$ & B.E.(MeV) & $r_{r m s}(\mathrm{fm})$ \\
\hline$\left[\Sigma_{Q} \Xi_{Q}^{\prime}\right]_{S}^{I=1 / 2}$ & & $\times$ & & & $\times$ & \\
\hline \multirow{3}{*}[\Sigma_{Q}\Xi_{Q}^{\prime}]{$_{S}^{I=3 / 2}$} & 0.80 & 3.54 & 1.97 & 0.80 & 25.63 & 0.74 \\
\hline & 0.90 & 14.53 & 1.14 & 0.90 & 53.38 & 0.57 \\
\hline & 1.00 & 67.46 & 0.67 & 1.00 & 156.78 & 0.40 \\
\hline \multirow{3}{*}[\Xi_{Q}^{\prime}\Omega_{Q}]{$_{S}^{I=1 / 2}$} & 0.90 & 28.50 & 0.88 & 0.90 & 80.49 & 0.49 \\
\hline & 0.95 & 29.65 & 0.86 & 0.95 & 80.91 & 0.49 \\
\hline & 1.00 & 44.09 & 0.75 & 1.00 & 107.01 & 0.45 \\
\hline \multirow{3}{*}[\Sigma_{Q}\Omega_{Q}]{$_{S}^{I=1}$} & 1.00 & 0.56 & 3.93 & 0.90 & 5.12 & 1.19 \\
\hline & 1.20 & 13.26 & 1.09 & 1.00 & 18.07 & 0.77 \\
\hline & 1.40 & 36.77 & 0.75 & 1.10 & 36.67 & 0.60 \\
\hline \multirow{3}{*}[\Xi_{Q}\Lambda_{Q}]{$_{S}^{I=1 / 2}$} & 1.10 & 1.91 & 2.47 & 0.90 & 10.33 & 0.96 \\
\hline & 1.30 & 3.03 & 2.03 & 1.20 & 28.65 & 0.67 \\
\hline & 1.50 & 2.87 & 2.08 & 1.50 & 27.78 & 0.68 \\
\hline \multirow{3}{*}[\Sigma_{Q}\Lambda_{Q}]{$_{S}^{I=1}$} & 0.90 & 0.28 & 5.48 & 0.80 & 0.34 & 5.19 \\
\hline & 1.00 & 14.81 & 1.09 & 0.90 & 16.02 & 0.79 \\
\hline & 1.10 & 47.34 & 0.73 & 1.00 & 62.13 & 0.52 \\
\hline \multirow{3}{*}[\Sigma_{Q}\Xi_{Q}]{$_{S}^{I=1 / 2}$} & 1.00 & 4.13 & 1.77 & 0.90 & 11.85 & 0.90 \\
\hline & 1.30 & 20.99 & 0.95 & 1.00 & 32.21 & 0.64 \\
\hline & 1.50 & 26.92 & 0.86 & 1.10 & 49.64 & 0.55 \\
\hline \multirow{3}{*}[\Sigma_{Q}\Xi_{Q}]{$_{S}^{I=3 / 2}$} & 0.90 & 1.29 & 2.91 & 0.90 & 19.88 & 0.78 \\
\hline & 1.00 & 9.15 & 1.32 & 0.95 & 29.69 & 0.68 \\
\hline & 1.10 & 33.32 & 0.82 & 1.00 & 46.46 & 0.58 \\
\hline \multirow{3}{*}[\Xi_{Q}^{\prime}\Lambda_{Q}]{$_{S}^{I=1 / 2}$} & 0.90 & 0.58 & 3.99 & 0.90 & 16.62 & 0.83 \\
\hline & 1.00 & 7.08 & 1.47 & 0.95 & 26.45 & 0.71 \\
\hline & 1.10 & 24.23 & 0.93 & 1.00 & 40.88 & 0.61 \\
\hline \multirow{3}{*}[\Xi_{Q}^{\prime}\Xi_{Q}]{$_{S}^{I=0}$} & 0.95 & 6.67 & 1.48 & 0.90 & 5.67 & 1.19 \\
\hline & 1.00 & 23.80 & 0.92 & 0.94 & 28.80 & 0.67 \\
\hline & 1.05 & 44.48 & 0.74 & 1.00 & 73.68 & 0.49 \\
\hline \multirow{3}{*}[\Xi_{Q}^{\prime}\Xi_{Q}]{$_{S}^{I=1}$} & 0.90 & 8.18 & 1.38 & 0.90 & 40.88 & 0.61 \\
\hline & 1.00 & 22.78 & 0.95 & 0.95 & 53.05 & 0.56 \\
\hline & 1.10 & 56.04 & 0.69 & 1.00 & 73.19 & 0.50 \\
\hline \multirow{3}{*}[\Xi_{Q}\Omega_{Q}]{$_{S}^{I=1 / 2}$} & 0.90 & 2.19 & 2.31 & 0.80 & 1.44 & 2.06 \\
\hline & 0.95 & 12.13 & 1.19 & 0.90 & 20.88 & 0.78 \\
\hline & 1.00 & 30.45 & 0.86 & 0.94 & 40.58 & 0.62 \\
\hline \multirow{3}{*}[\Lambda_{Q}\Omega_{Q}]{$_{S}^{I=0}$} & 1.00 & 5.40 & 1.60 & 0.90 & 14.10 & 0.86 \\
\hline & 1.10 & 16.55 & 1.04 & 1.00 & 36.07 & 0.62 \\
\hline & 1.20 & 32.02 & 0.82 & 1.10 & 64.89 & 0.51 \\
\hline
\end{tabular}


TABLE IX: The numerical results for the spin-triplet $(S=1)$ case with the OBE potential. $\times$ indicates no binding solutions exist.

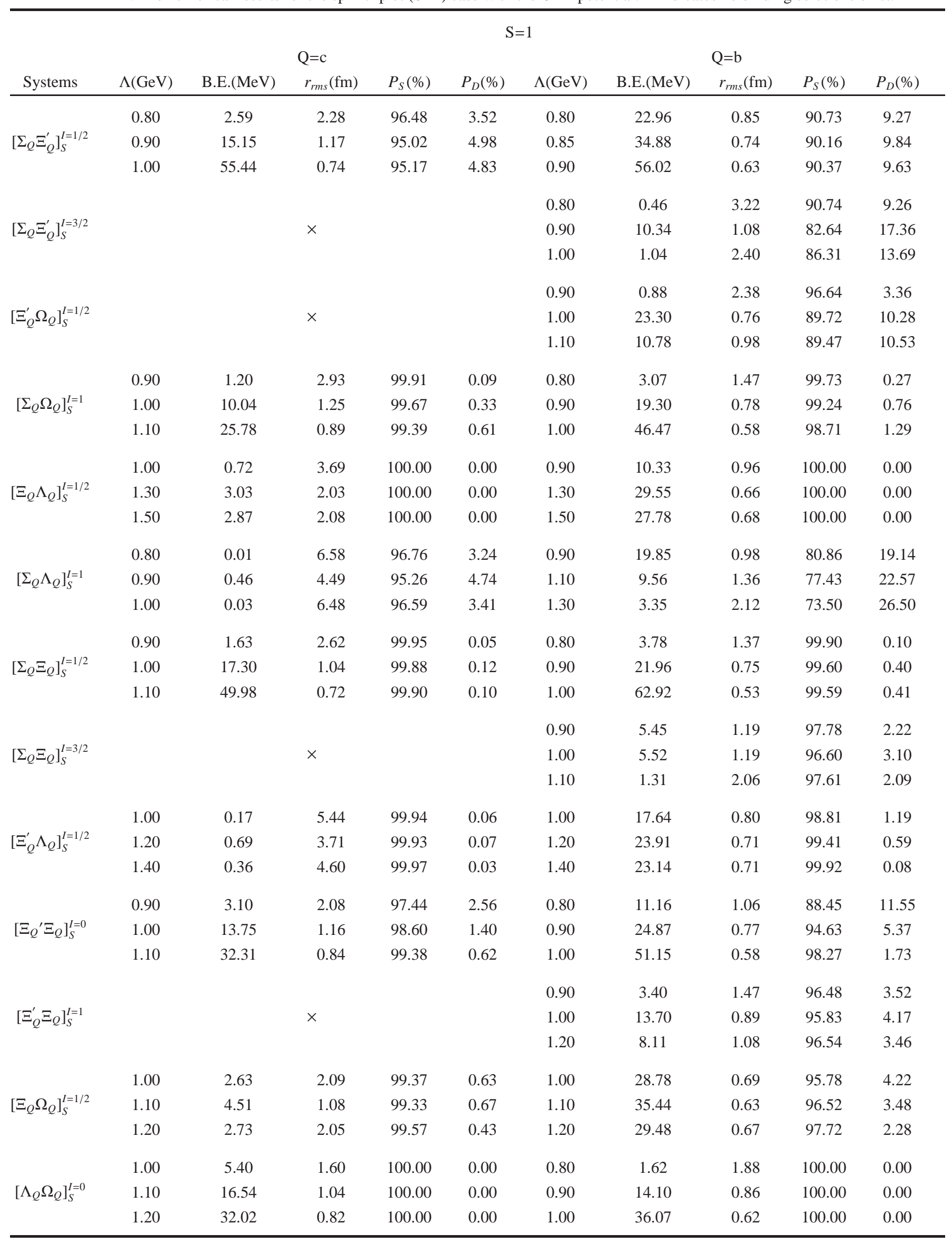



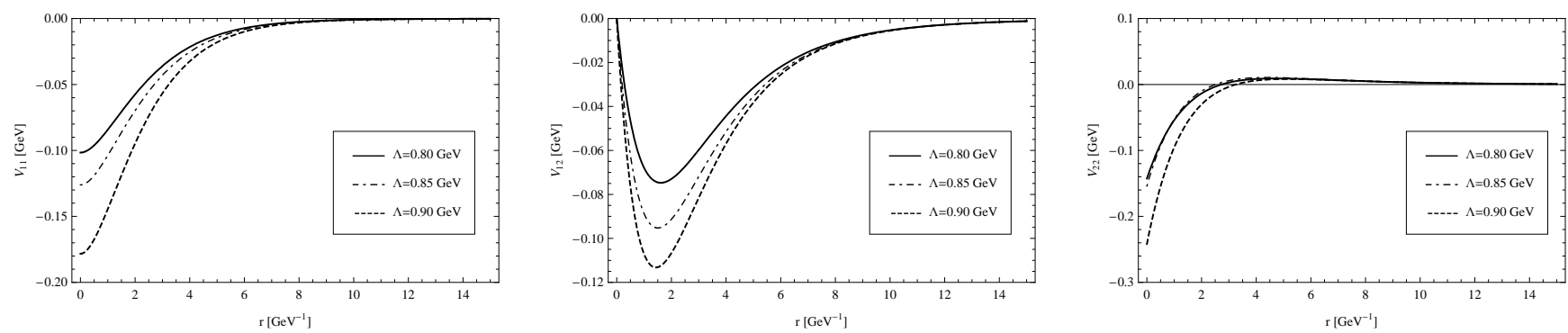

FIG. 4: The potential of system $\Sigma_{c} \Xi_{c}^{\prime}\left[I\left(J^{P}\right)=\frac{1}{2}\left(1^{+}\right)\right]$within the OBE model. $V_{11}, V_{12}$ and $V_{22}$ are for the transitions ${ }^{3} S_{1} \leftrightarrow{ }^{3} S_{1},{ }^{3} S_{1} \leftrightarrow{ }^{3} D_{1}$ and ${ }^{3} D_{1} \leftrightarrow{ }^{3} D_{1}$, respectively.

\section{A. Numerical Results for The $\Lambda_{Q} \Lambda_{Q}$ System with The OPE Potential}

The state $\Lambda_{Q} \Lambda_{Q}(\mathrm{Q}=\mathrm{b}, \mathrm{c})$ is the heavy analogue of the $\mathrm{H}$ dibaryon. We use the $\pi$ exchange potential and include the coupledchannel effect. Actually, the pion exchange is forbidden for the $\Lambda_{Q} \Lambda_{Q}$ system due to the isospin conservation of the strong interaction. The binding solution is mainly due to the coupled-channel effect. We give the numerical results in Table $X$ and plot the dependence of the binding energy on the cutoff parameter in Fig. 10.

$\Lambda_{Q} \Lambda_{Q}\left[I\left(J^{P}\right)=0\left(0^{+}\right)\right]$. We reproduce the numerical results of Ref. [20] for the state $\Lambda_{c} \Lambda_{c}\left({ }^{1} S_{0}\right)$, and list them in Table X We also extend the same formalism to the bottomed sector. A bound state $\Lambda_{b} \Lambda_{b}\left({ }^{1} S_{0}\right)$ with binding energy $9.09 \sim 26.99 \mathrm{MeV}$ appears when the cutoff parameter is chosen between $0.80 \mathrm{GeV}$ and $0.90 \mathrm{GeV}$. Correspondingly, the root-mean-square radius varies from $1.08 \mathrm{fm}$ to $0.77 \mathrm{fm}$. However, if the cutoff parameter increases to $1.10 \mathrm{GeV}$, the binding energy will reach as high as 105.17 MeV. There are five channels shown in Table IV for this state. The $\Lambda_{b} \Lambda_{b}\left({ }^{1} S_{0}\right)$ component is dominant with a probability about $90 \%$. The contribution of the components $\Sigma_{b} \Sigma_{b}\left({ }^{1} S_{0}\right)$ and $\Sigma_{b}^{*} \Sigma_{b}^{*}\left({ }^{1} S_{0}\right)$ is very small, around $1 \%$.

$\Lambda_{Q} \Lambda_{Q}\left[I\left(J^{P}\right)=0\left(0^{-}\right)\right]$. We also extend the same analysis to the case with the orbital excitation $L=1$ and obtain a loosely bound state of $\Lambda_{c} \Lambda_{c}\left[0\left(0^{-}\right)\right]$with binding energy $0.19 \sim 24.97 \mathrm{MeV}$ when the cutoff parameter is chosen between $1.36 \mathrm{GeV}$ and $1.45 \mathrm{GeV}$. The binding energy will increase to $99.26 \mathrm{MeV}$ when the cutoff parameter is $1.60 \mathrm{GeV}$. The contribution of the dominant channel, $\Lambda_{c} \Lambda_{c}\left({ }^{3} P_{0}\right)$, is $90.73 \% \sim 81.72 \%$ when the cutoff parameter is around $1.36 \sim 1.45 \mathrm{GeV}$. The channel $\Sigma_{c}^{*} \Sigma_{c}^{*}\left({ }^{3} P_{0}\right)$ provides a fairly small contribution, less than $1 \%$. For the corresponding bottomed state, its binding energy is $0.50 \sim$ $34.63 \mathrm{MeV}$ when the cutoff parameter is $0.95 \sim 1.10 \mathrm{GeV}$, which may also be a good molecule candidate.

$\Lambda_{Q} \Lambda_{Q}\left[I\left(J^{P}\right)=0\left(1^{-}\right)\right]$. We consider seven channels in this case, which are listed in Table IV. We obtain a shallow bound state with binding energy $0.91 \sim 20.27 \mathrm{MeV}$ in the charmed sector when the cutoff parameter is $1.45 \sim 1.50 \mathrm{GeV}$. And the contribution of the dominant channel, $\Lambda_{c} \Lambda_{c}\left({ }^{3} P_{1}\right)$, is $79.15 \% \sim 67.55 \%$. If the cutoff parameter increases to $1.60 \mathrm{GeV}$, the binding energy will reach $79.78 \mathrm{MeV}$. The channel with the second largest contribution is $\Sigma_{c} \Sigma_{c}\left({ }^{3} P_{1}\right)$, with a probability of $14.55 \% \sim 22.90 \%$ when the cutoff parameter is around $1.45 \sim 1.50 \mathrm{GeV}$. However, the probabilities of the other three channels, $\Sigma_{c} \Sigma_{c}^{*}\left({ }^{3} P_{1}\right), \Sigma_{c} \Sigma_{c}^{*}\left({ }^{5} P_{1}\right)$ and $\Sigma_{c} \Sigma_{c}^{*}\left({ }^{5} F_{1}\right)$, are tiny as shown in Table $\mathrm{X}$ The situation of the bottomed case is similar to that of the charmed case except that the binding of the former is deeper.

\section{B. Numerical Results for The $\Lambda_{Q} \Lambda_{Q}$ System with The OBE Potential}

In this subsection, we investigate the $\Lambda_{Q} \Lambda_{Q}$ system with the OBE potential which not only includes the long-range $\pi$ exchange interaction but also the medium-/short-range $\eta, \sigma, \rho$ and $\omega$ exchange interaction. The numerical results are shown in Table XI.

We obtain a weakly bound state for $\Lambda_{c} \Lambda_{c}\left[I\left(J^{P}\right)=0\left(0^{+}\right)\right]$. The binding energy is $2.53 \sim 55.11 \mathrm{MeV}$ when the cutoff parameter is around $0.80 \sim 1.00 \mathrm{GeV}$. Accordingly its root-mean-square radius is about $2.31 \sim 0.73$ fm, which is comparable with the size of the deuteron. Similar to the OPE potential case, the $\Lambda_{c} \Lambda_{c}\left({ }^{1} S_{0}\right)$ component is dominant with a probability about $98.69 \% \sim 86.79 \%$, and the total contributions of the other channels are less than $15 \%$. For the state $\Lambda_{b} \Lambda_{b}\left[0\left(0^{+}\right)\right]$, the binding energy is much larger as expected. Its binding energy is $27.30 \mathrm{MeV}$ when the cutoff parameter is $0.80 \mathrm{MeV}$. When the cutoff parameter is $1.00 \mathrm{GeV}$, the binding energy reaches as high as $148.17 \mathrm{MeV}$.

For the state $\Lambda_{c} \Lambda_{c}\left[I\left(J^{P}\right)=0\left(0^{-}\right)\right]$, the binding energy is $4.69 \mathrm{MeV}$ when the cutoff parameter is $1.15 \mathrm{GeV}$. When we increase the cutoff parameter to $1.25 \mathrm{GeV}$, the binding energy is $61.36 \mathrm{MeV}$. The probability of the dominant channel $\Lambda_{c} \Lambda_{c}\left({ }^{3} P_{0}\right)$ is about $85.12 \% \sim 71.08 \%$. The contribution of the second dominant channel is $\Sigma_{c} \Sigma_{c}^{*}\left({ }^{3} P_{0}\right)$, with a probability about $11.48 \% \sim 23.57 \%$. The results of the bottomed state $\Lambda_{b} \Lambda_{b}\left[0\left(0^{-}\right)\right]$are similar to those of the charmed case, but with deeper binding energy. The binding energy is $2.80 \sim 100.54 \mathrm{MeV}$ with the cutoff parameter around $0.85 \sim 1.05 \mathrm{GeV}$. The probabilities of the channels 
TABLE X: The binding solutions of $\Lambda_{Q} \Lambda_{Q}$ with OPE potential. " $\Lambda$ " is the cutoff parameter. "B.E." and " $r_{r m s}$ " are the binding energy and the root-mean-square radius, respectively. " $P_{i}$ " is the probability of the individual channel which are given in Table IV

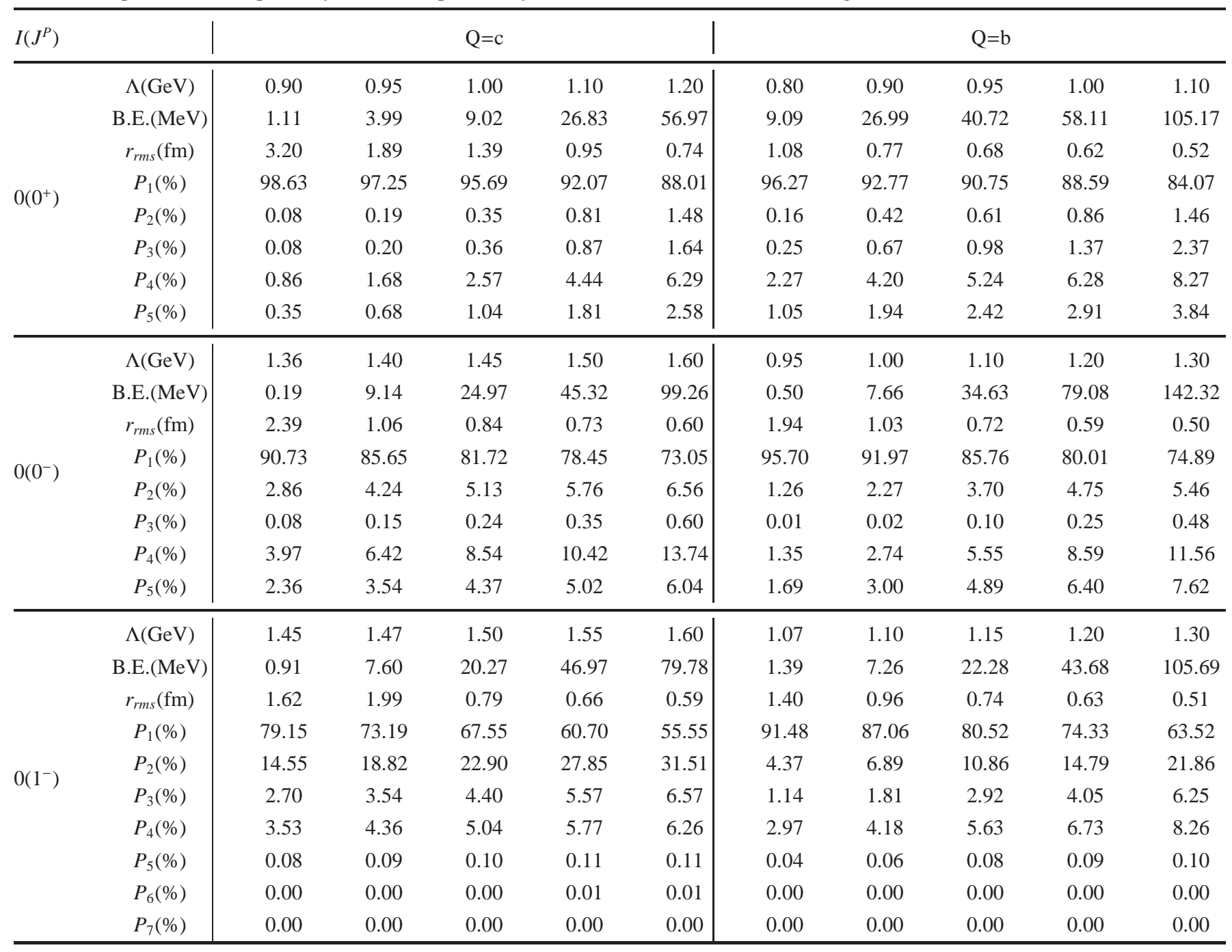

$\Lambda_{b} \Lambda_{b}\left({ }^{3} P_{1}\right)$ and $\Sigma_{b} \Sigma_{b}^{*}\left({ }^{3} P_{1}\right)$ are about $95.93 \% \sim 73.92 \%$ and $1.45 \% \sim 18.90 \%$ respectively.

The state $\Lambda_{c} \Lambda_{c}\left[I\left(J^{p}\right)=0\left(1^{-}\right)\right]$with binding energy around $1.35 \sim 65.05 \mathrm{MeV}$ and cutoff parameter around $1.16 \sim 1.25 \mathrm{GeV}$ may also be a loosely bound state. But the binding solutions depend sensitively on the cutoff parameter. The binding energy of the state $\Lambda_{b} \Lambda_{b}\left[0\left(1^{-}\right)\right]$is $0.24 \sim 74.65 \mathrm{MeV}$ when the cutoff parameter is $0.90 \sim 1.05 \mathrm{GeV}$.

Besides the transition induced by the OPE force in the flavor space, we have also considered the transitions caused by the eta meson and rho/omega meson exchange, which greatly enhances the non-diagonal matrix element in the Hamiltonian. With the same cutoff parameter, we can clearly see that the binding energy in the OBE case is larger than that in the OPE case. For example, the binding energy for the $\Lambda_{c} \Lambda_{c}\left[I\left(J^{P}\right)=0\left(0^{+}\right)\right]$state is $1.11 \mathrm{MeV}$ in the OPE case if one fixes the cutoff at $0.90 \mathrm{GeV}$. However, the binding energy increase to $16.61 \mathrm{MeV}$ in the OBE case with the same cutoff. In other words, the medium- and short-range attractive force plays a significant role in the formation of the loosely bound $\Lambda_{c} \Lambda_{c}$ and $\Lambda_{b} \Lambda_{b}$ states.

\section{DISCUSSIONS AND CONCLUSIONS}

We have investigated the possible deuteron-like molecules composed of two heavy flavor baryons with the form of " $A_{Q} B_{Q}$ ". We have also performed an extensive analysis of the $\Lambda_{Q} \Lambda_{Q}(\mathrm{Q}=\mathrm{b}, \mathrm{c})$ system, which is the heavy analogue of the $\mathrm{H}$ dibaryon.

The weakly bound states are usually very sensitive to potential details including the coupling constants and form factors etc. Sometimes small change of the coupling constants may dismantle the bound state.

Throughout this work, we have adopted the root-mean-square radius and binding energy of the system to judge whether the 
TABLE XI: The binding solutions of $\Lambda_{Q} \Lambda_{Q}$ with the OBE potential. " $\Lambda$ " is the cutoff parameter. "B.E." and " $r_{r m s}$ " are the binding energy and the root-mean-square radius, respectively. " $P_{i}$ " is the probability of the individual channel which are given in Table IV

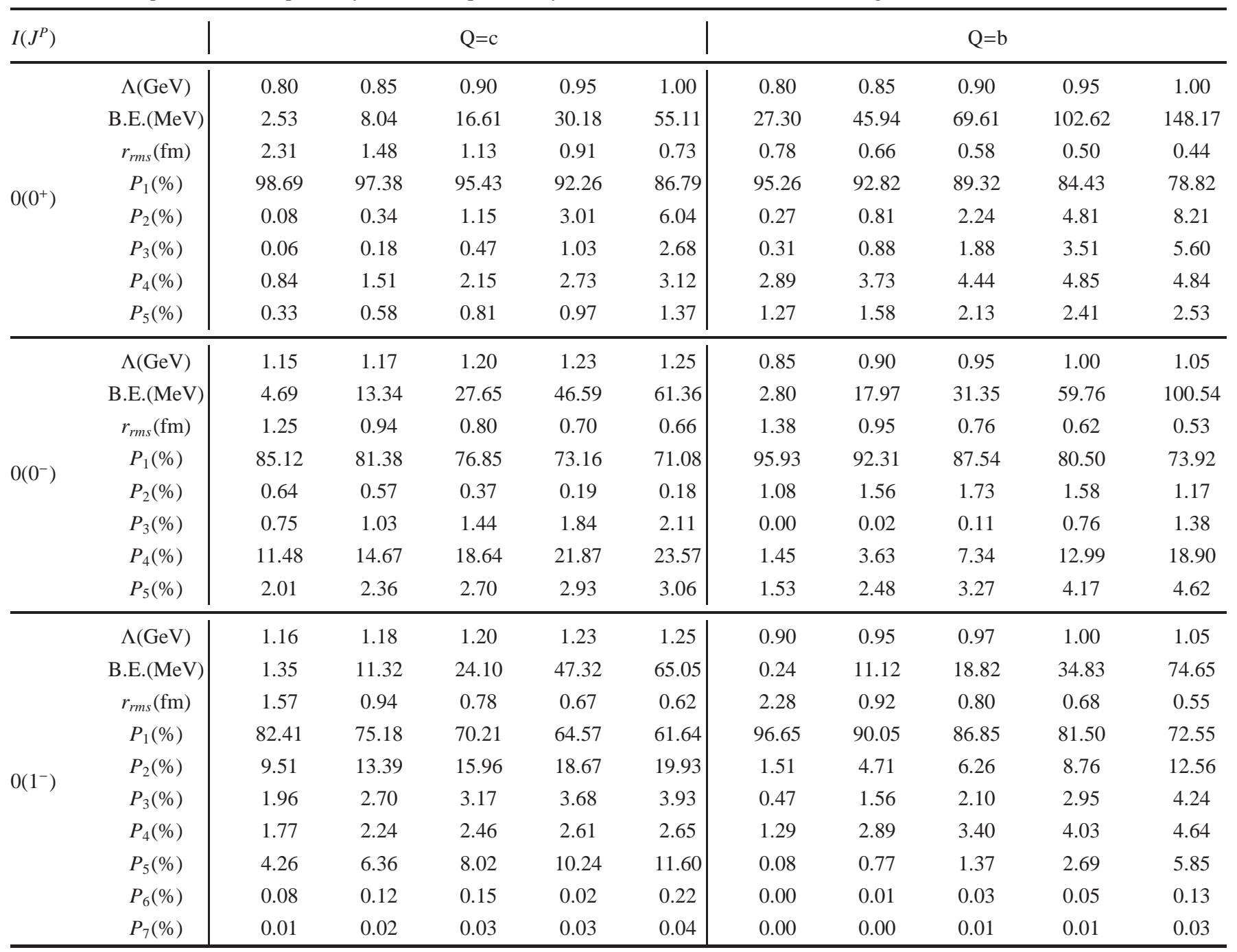

system is a loosely bound molecular state. There exists another intuitive approach. The relative momentum of the loosely bound system $p \sim \sqrt{2 \mu E}$ probes distance around $\frac{1}{p}$, where $\mu$ is the reduced mass and $E$ is the binding energy. For a loosely bound state, $\frac{1}{p}$ should be much larger than the interaction range of the potential, which is around $\frac{1}{m_{\rho, \omega, \sigma}} \sim(0.2-0.3) \mathrm{fm}$. In other words, the size of the system should be larger than $(0.6 \sim 1.0) \mathrm{fm}$. Accordingly, the binding energy should be much smaller than $\frac{m_{\rho}^{2}}{M_{B}}$ where $M_{B}$ is the charmed or bottomed baryon mass. Numerically, the binding energy should be much less than $240 \mathrm{MeV}$ and $100 \mathrm{MeV}$ for the charmed and bottomed systems respectively. In other words, those states in Tables VII] XI which do not satisfy the above criteria should not be regarded as the loosely bound molecular states.

For the spin-singlet systems with the " $A_{Q} B_{Q}$ " form, the pion exchange force is not strong enough to form bound states for the five systems, $\left[\Sigma_{Q} \Xi_{Q}^{\prime}\right]_{S=0}^{(I=1 / 2,3 / 2)},\left[\Sigma_{Q} \Lambda_{Q}\right]_{S=0}^{I=1}$ and $\left[\Xi_{Q} \Xi_{Q}^{\prime}\right]_{S=0}^{(I=0,1)}(\mathrm{Q}=\mathrm{b}, \mathrm{c})$. When we add the contributions from the scalar and vector meson exchanges, some bound states appear. The following five states $\left[\Sigma_{c} \Omega_{c}\right]_{S=0}^{I=1}$, $\left[\Xi_{c} \Lambda_{c}\right]_{S=0}^{I=1 / 2},\left[\Xi_{b} \Lambda_{b}\right]_{S=0}^{I=1 / 2},\left[\Sigma_{c} \Xi_{c}\right]_{S=0}^{I=1 / 2}$ and $\left[\Lambda_{c} \Omega_{c}\right]_{S=0}^{I=0}$ are all very loosely bound with small binding energies and large root-mean-square radii with cutoff parameter $1.0 \sim 1.50 \mathrm{GeV}$. They are good candidates of molecules.

In the spin-triplet case, the numerical results with the one-pion-exchange potential alone indicate that $\left[\Sigma_{Q} \Xi_{Q}^{\prime}\right]_{S=1}^{I=1 / 2}(\mathrm{Q}=\mathrm{b}, \mathrm{c})$, $\left[\Sigma_{Q} \Lambda_{Q}\right]_{S=1}^{I=1}(\mathrm{Q}=\mathrm{b}, \mathrm{c}),\left[\Sigma_{b} \Xi_{b}^{\prime}\right]_{S=1}^{I=3 / 2}$ and $\left[\Xi_{b} \Xi_{b}^{\prime}\right]_{S=1}^{I=0}$ may be loosely bound states. They have shallow binding solutions when the cutoff parameter is around $0.90 \sim 1.50 \mathrm{GeV}$. The three states $\left[\Sigma_{c} \Xi_{c}^{\prime}\right]_{S=1}^{I=3 / 2},\left[\Xi_{c} \Xi_{c}^{\prime}\right]_{S=1}^{I=0}$ and $\left[\Xi_{b} \Xi_{b}^{\prime}\right]_{S=1}^{I=1}$ do not have binding solutions until the cutoff parameter reaches $1.80 \mathrm{GeV}$. When taking the vector and scalar boson exchanges into account, the numerical results do not change significantly except that the bound state of $\left[\Sigma_{c} \Xi_{c}^{\prime}\right]_{S=1}^{I=3 / 2}$ disappear and the binding energy in some channels 
becomes deeper. Therefore, we conclude that the long-range one-pion-exchange interaction plays an dominant role in forming these bound states. Comparing the results of OPE model with those of the OBE model, one notices that the contribution of the D wave is smaller for the latter, which implies that the S-D mixing mainly comes from the pion exchange. Our results suggest that

the states $\left[\Xi_{Q}^{\prime} \Lambda_{Q}\right]_{S=1}^{I=1 / 2}(\mathrm{Q}=\mathrm{b}, \mathrm{c})$ and $\left[\Xi_{Q} \Omega_{Q}\right]_{S=1}^{I=1 / 2}(\mathrm{Q}=\mathrm{b}, \mathrm{c})$ with shallow binding solutions and reasonable cutoff parameter may also be good candidates of molecules.

For the heavy analogue of the $\mathrm{H}$ dibaryon, our results indicate that $\Lambda_{Q} \Lambda_{Q}(\mathrm{Q}=\mathrm{b}, \mathrm{c})$ with quantum numbers $I\left(J^{P}\right)=0\left(0^{+}\right), 0\left(0^{-}\right)$ and $0\left(1^{-}\right)$may all be molecules. The binding solutions of $\Lambda_{Q} \Lambda_{Q}$ system with the OPE potential mainly come from the coupledchannel effect. Besides the transition induced by the OPE force in the flavor space, we have also considered the transitions caused by the eta meson and rho/omega meson exchange. With the same cutoff parameter, the binding energy in the OBE case is larger than that in the OPE case. The medium- and short-range attractive force plays a significant role in the formation of the loosely bound $\Lambda_{c} \Lambda_{c}$ and $\Lambda_{b} \Lambda_{b}$ states.

The authors studied the $\Lambda_{Q} \Lambda_{Q}$ system at the quark level, and obtained bound states with mass $4516 \mathrm{MeV}$ for $\Lambda_{c} \Lambda_{c}$ and 9175 $\mathrm{MeV}$ for $\Lambda_{b} \Lambda_{b}$ [30]. Theoretical investigations of these molecular states with other phenomenological models are desirable.

If these states really exist as molecules, once produced, they will be very stable because this system decays via weak interaction. It is difficult to produce the states with double charm or double bottom experimentally. However, there is still hope to search for these interesting long-lived molecular states with double heavy flavor at facilities such as the Large Hadron Collider and RHIC.

All the molecule states (except those with $\Sigma_{Q}$ ) are very stable because their components have a long lifetime around $10^{-13} \sim$ $10^{-12} \mathrm{~s}$. On the other hand, the width of $\Sigma_{c}$ is about $2.2 \mathrm{MeV}$ [28], this narrow width ensures relatively long lifetime for the " $\Sigma_{c} X$ "type molecules. Such states can decay into $X \Lambda_{c}^{+} \pi$ followed by $\Lambda_{c}^{+} \rightarrow p K^{-} \pi^{+}$. For the bottomed case, $\Sigma_{b} X \rightarrow X \Lambda_{b}^{0} \pi$ followed by $\Lambda_{b}^{0} \rightarrow \Lambda_{c}^{+} \pi^{-}$and $\Lambda_{c}^{+} \rightarrow p K^{-} \pi^{+}$. These decay modes may be helpful to search for such states in the future experiment.

\section{Acknowledgments}

One of the authors (N. L.) is very grateful to Z. G. Luo and Dr. Y. R. Liu for very helpful discussions. This project was supported by the National Natural Science Foundation of China under Grants 11075004, 11021092 and Ministry of Science and Technology of China (2009CB825200).

[1] S. K. Choi et al. [Belle Collaboration], Phys. Rev. Lett. 91, 262001 (2003); V. M. Abazov et al. [D0 Collaboration], Phys. Rev. Lett. 93, 162002 (2004); D. E. Acosta et al. [CDF II Collaboration], Phys. Rev. Lett. 93, 072001 (2004); B. Aubert et al. [BABAR Collaboration], Phys. Rev. D 71, 071103 (2005).

[2] P. Pakhlov et al. [Belle Collaboration], Phys. Rev. Lett. 100, 202001 (2008).

[3] B. Aubert et al. [BABAR Collaboration], Phys. Rev. Lett. 95, 142001 (2005); C. Z. Yuan et al. [Belle Collaboration], Phys. Rev. Lett. 99, 182004 (2007).

[4] S. K. Choi et al. [Belle Collaboration], Phys. Rev. Lett. 100, 142001 (2008).

[5] B. Collaboration, arXiv:1105.4583 [hep-ex].

[6] M. B. Voloshin and L. B. Okun, Pisma Zh. Eksp. Teor. Fiz. 23, 369 (1976)[JETP Lett. 23, 333 (1976)].

[7] A. De Rujula, H. Georgi and S. L. Glashow, Phys. Rev. Lett. 38, 317 (1977).

[8] N. A. Törnqvist, Nuovo Cim. A 107, 2471 (1994).

[9] N. A. Törnqvist, Z. Phys. C 61, 525 (1994).

[10] X. Liu, Z. G. Luo, Y. R. Liu and S. L. Zhu, Eur. Phys. J. C 61, 411 (2009).

[11] G. J. Ding, J. F. Liu and M. L. Yan, Phys. Rev. D 79, 054005 (2009).

[12] Z. -F. Sun, J. He, X. Liu, Z. -G. Luo and S. -L. Zhu, Phys. Rev. D 84, 054002 (2011).

[13] F. Fröemel, B. Juliá-Díaz and D. O. Riska, Nucl. Phys. A 750, 337 (2005).

[14] B. Juliá-Díaz and D. O. Riska, Nucl. Phys. A 755, 431 (2005).

[15] R. L. Jaffe, Phys. Rev. Lett. 38, 195 (1977) [Erratum-ibid. 38, 617 (1977)].

[16] S. R. Beane et al. (NPLQCD Collaboration), Phys. Rev. Lett. 106, 162001 (2011); T. Inoue et al. (HAL QCD Collaboration), Phys. Rev. Lett. 106, 162002 (2011); (HAL QCD Collaboration), Nucl. Phys. A881, 28 (2012); (for HAL QCD Collaboration), Proc. Sci. LATTICE2011 (2011) 124.

[17] J. Haidenbauer and U. G. Meißner, Phys. Lett. B 706, 100 (2011); Nucl. Phys. A881, 44 (2012).

[18] M. Chen, H. Huang, J. Ping and F. Wang, Phys. Rev. C 83, 015202 (2011).

[19] N. Lee, Z. G. Luo, X. L. Chen and S. L. Zhu, Phys. Rev. D 84, 014031 (2011).

[20] W. Meguro, Y. -R. Liu and M. Oka, Phys. Lett. B 704, 547 (2011)

[21] N. A. Törnqvist, Phys. Rev. Lett. 67, 556 (1991).

[22] G. G. Simon, C. Schmitt and V. H. Walther, Nucl. Phys. A 364 (1981) 285. 
[23] T. M. Yan, H. Y. Cheng, C. Y. Cheung, G. L. Lin, Y. C. Lin and H. L. Yu, Phys. Rev. D 46, 1148 (1992) [Erratum-ibid. D 55, 5851 (1997)].

[24] D. O. Riska and G. E. Brown, Nucl. Phys. A 679, 577 (2001).

[25] R. Machleidt, Phys. Rev. C 63, 024001 (2001).

[26] X. Cao, B. S. Zou and H. S. Xu, Phys. Rev. C 81, 065201 (2010).

[27] A. Calle Cordon and E. Ruiz Arriola, Phys. Rev. C 81, 044002 (2010).

[28] K. Nakamura, et al. (Particle Data Group), J. Phys. G 37, 075021 (2010).

[29] A. G. Abrashkevich, D. G. Abrashkevich, M. S. Kaschiev and I. V. Puzynin, Comput. Phys. Commun. 85 65-81 (1995).

[30] S. M. Gerasyuta and E. E. Matskevich, Int. J. Mod. Phys. E21 1250058 (2012).

\section{APPENDIX}

\section{A. Some Helpful Functions}

The functions $H_{i}$ etc are defined as,

$$
\begin{aligned}
& H_{0}(\Lambda, m, r)=Y(u r)-\frac{\lambda}{u} Y(\lambda r)-\frac{r \beta^{2}}{2 u} Y(\lambda r), \quad H_{1}(\Lambda, m, r)=Y(u r)-\frac{\lambda}{u} Y(\lambda r)-\frac{r \lambda^{2} \beta^{2}}{2 u^{3}} Y(\lambda r), \\
& H_{2}(\Lambda, m, r)=Z_{1}(u r)-\frac{\lambda^{3}}{u^{3}} Z_{1}(\lambda r)-\frac{\lambda \beta^{2}}{2 \mu^{3}} Y(\lambda r), \quad H_{3}(\Lambda, m, r)=Z(u r)-\frac{\lambda^{3}}{u^{3}} Z(\lambda r)-\frac{\lambda \beta^{2}}{2 u^{3}} Z_{2}(\lambda r), \\
& M_{0}(\Lambda, m, r)=-\frac{1}{\theta r}\left[\cos (\theta r)-e^{-\lambda r}\right]+\frac{\beta^{2}}{2 \theta \lambda} e^{-\lambda r}, \quad M_{1}(\Lambda, m, r)=-\frac{1}{\theta r}\left[\cos (\theta r)-e^{-\lambda r}\right]-\frac{\lambda \beta^{2}}{2 \theta^{3}} e^{-\lambda r}, \\
& M_{3}(\Lambda, m, r)=-\left[\cos (\theta r)-\frac{3 \sin (\theta r)}{\theta r}-\frac{3 \cos (\theta r)}{\theta^{2} r^{2}}\right] \frac{1}{\theta r}-\frac{\lambda^{3}}{\theta^{3}} Z(\lambda r)-\frac{\lambda \beta^{2}}{2 \theta^{3}} Z_{2}(\lambda r),
\end{aligned}
$$

where,

$$
\beta^{2}=\Lambda^{2}-m^{2}, \quad u^{2}=m^{2}-Q_{0}^{2}, \quad \theta^{2}=-\left(m^{2}-Q_{0}^{2}\right), \quad \lambda^{2}=\Lambda^{2}-Q_{0}^{2},
$$

and

$$
Y(x)=\frac{e^{-x}}{x}, \quad Z(x)=\left(1+\frac{3}{x}+\frac{3}{x^{2}}\right) Y(x), \quad Z_{1}(x)=\left(\frac{1}{x}+\frac{1}{x^{2}}\right) Y(x), \quad Z_{2}(x)=(1+x) Y(x) .
$$

Fourier transformation formulae read:

$$
\begin{aligned}
& \frac{1}{u^{2}+Q^{2}} \rightarrow \frac{u}{4 \pi} H_{0}(\Lambda, m, r), \quad \frac{Q^{2}}{u^{2}+Q^{2}} \rightarrow-\frac{u^{3}}{4 \pi} H_{1}(\Lambda, m, r), \\
& \frac{Q}{u^{2}+Q^{2}} \rightarrow \frac{i u^{3}}{4 \pi} r H_{2}(\Lambda, m, r), \quad \frac{Q_{i} Q_{j}}{u^{2}+Q^{2}} \rightarrow-\frac{u^{3}}{12 \pi}\left[H_{3}(\Lambda, m, r) k_{i j}+H_{1}(\Lambda, m, r) \delta_{i j}\right],
\end{aligned}
$$

where, $k_{i j}=3 \frac{r_{i} r_{j}}{r^{2}}-\delta_{i j}$. If the form factor is not introduced, there will be delta terms in the second and fourth formule. In the above expressions, we employ another function, $-\frac{u^{3}}{4 \pi}\left[H_{1}(\Lambda, m, r)-H_{0}(\Lambda, m, r)\right]$, to substitute for the delta term. If one neglects the delta term, one should adopt the following formulae,

$$
\frac{Q^{2}}{u^{2}+Q^{2}} \rightarrow-\frac{u^{3}}{4 \pi} H_{0}(\Lambda, m, r), \quad \frac{Q_{i} Q_{j}}{u^{2}+Q^{2}} \rightarrow-\frac{u^{3}}{12 \pi}\left[H_{3}(\Lambda, m, r) k_{i j}+H_{0}(\Lambda, m, r) \delta_{i j}\right] .
$$

If $u^{2}=m_{e x}^{2}-Q_{0}^{2}<0$, the last formula of the Eq. (27) should be

$$
\frac{Q_{i} Q_{j}}{u^{2}+Q^{2}} \rightarrow-\frac{\theta^{3}}{12 \pi}\left[M_{3}(\Lambda, m, r) k_{i j}+M_{1}(\Lambda, m, r) \delta_{i j}\right] .
$$

Accordingly, we make the replacement $M_{1}(\Lambda, m, r) \rightarrow M_{0}(\Lambda, m, r)$ to neglect the delta term.

\section{B. The Numerical Results of The " $A_{Q} B_{Q}$ " Systems When The Contact Term Is Included}

For comparison, we collect the numerical results of the " $A_{Q} B_{Q}$ " systems in Tables XII and XIII when the contact term is included. 
TABLE XII: The binding solutions of the spin-singlet " $A_{Q} B_{Q}$ " systems when the interaction potential includes the contact term.

\begin{tabular}{|c|c|c|c|c|c|c|}
\hline \multirow[b]{3}{*}{ States } & \multicolumn{6}{|c|}{$S=0$} \\
\hline & \multicolumn{3}{|c|}{$Q=c$} & \multicolumn{3}{|c|}{$Q=b$} \\
\hline & $\Lambda(\mathrm{GeV})$ & B.E.(MeV) & $r_{r m s}(\mathrm{fm})$ & $\Lambda(\mathrm{GeV})$ & B.E.(MeV) & $r_{r m s}(\mathrm{fm})$ \\
\hline \multirow{3}{*}[\Sigma_{Q}\Xi_{Q}^{\prime}]{$_{S}^{I=\frac{1}{2}}$} & 0.80 & 52.87 & 0.64 & 0.80 & 162.52 & 0.34 \\
\hline & 0.85 & 82.54 & 0.55 & 0.85 & 219.83 & 0.30 \\
\hline & 0.90 & 137.34 & 0.45 & 0.90 & 321.82 & 0.26 \\
\hline \multirow{3}{*}[\Sigma_{Q}\Xi_{Q}^{\prime}]{$_{S}^{I=\frac{3}{2}}$} & 1.30 & 0.23 & 5.44 & 1.00 & 0.45 & 3.49 \\
\hline & 1.40 & 1.48 & 3.03 & 1.10 & 0.45 & 3.49 \\
\hline & 1.50 & 4.11 & 2.10 & 1.20 & 4.61 & 1.66 \\
\hline \multirow[t]{3}{*}[\Xi_{Q}^{\prime}\Omega_{Q}]{$_{S}^{I=\frac{1}{2}}$} & 1.10 & 0.94 & 4.56 & 0.90 & 1.12 & 2.39 \\
\hline & 1.20 & 1.03 & 3.29 & 1.00 & 5.36 & 1.41 \\
\hline & 1.30 & 2.53 & 2.35 & 1.10 & 7.59 & 1.32 \\
\hline \multirow[t]{3}{*}[\Sigma_{Q}\Omega_{Q}]{$_{S}^{I=1}$} & 0.90 & 2.55 & 2.08 & 0.80 & 4.89 & 1.18 \\
\hline & 0.95 & 8.80 & 1.26 & 0.85 & 15.30 & 0.78 \\
\hline & 1.00 & 18.69 & 0.94 & 0.90 & 31.25 & 0.61 \\
\hline \multirow{3}{*}[\Xi_{Q}\Lambda_{Q}]{$_{S}^{I=\frac{1}{2}}$} & 1.00 & 0.73 & 3.67 & 0.80 & 0.55 & 2.94 \\
\hline & 1.10 & 1.77 & 2.55 & 0.85 & 4.93 & 1.25 \\
\hline & 1.20 & 2.31 & 2.28 & 0.90 & 10.39 & 0.96 \\
\hline \multirow{3}{*}[\Sigma_{Q}\Lambda_{Q}]{$_{S=0}^{I=1}$} & 1.80 & 0.21 & 5.96 & 0.80 & 0.13 & 6.53 \\
\hline & 1.90 & 0.83 & 3.99 & 1.00 & 0.19 & 6.06 \\
\hline & 2.00 & 2.00 & 2.69 & 1.20 & 0.32 & 5.35 \\
\hline \multirow[t]{3}{*}[\Sigma_{Q}\Xi_{Q}]{$_{S}^{I=\frac{1}{2}}$} & 0.90 & 2.59 & 2.12 & 0.80 & 6.13 & 1.08 \\
\hline & 0.95 & 10.29 & 1.22 & 0.85 & 14.88 & 0.81 \\
\hline & 1.00 & 24.38 & 0.88 & 0.90 & 30.65 & 0.63 \\
\hline \multirow[t]{3}{*}[\Sigma_{Q}\Xi_{Q}]{$_{S}^{I=\frac{3}{2}}$} & 1.50 & 0.42 & 4.55 & 0.85 & 0.85 & 2.56 \\
\hline & 1.60 & 1.28 & 3.06 & 1.00 & 2.25 & 1.82 \\
\hline & 1.70 & 2.66 & 2.30 & 1.20 & 3.69 & 1.61 \\
\hline \multirow[t]{3}{*}[\Xi_{Q}^{\prime}\Lambda_{Q}]{$_{S}^{I=\frac{1}{2}}$} & 1.10 & 0.45 & 4.39 & 0.85 & 1.55 & 2.00 \\
\hline & 1.20 & 1.02 & 3.32 & 1.00 & 7.16 & 1.16 \\
\hline & 1.30 & 1.86 & 2.70 & 1.20 & 11.56 & 1.08 \\
\hline \multirow[t]{3}{*}[\Xi_{Q}^{\prime}\Xi_{Q}]{$_{S}^{I=0}$} & 1.10 & 1.85 & 2.59 & 1.00 & 2.51 & 1.65 \\
\hline & 1.20 & 6.37 & 1.60 & 1.10 & 11.11 & 1.05 \\
\hline & 1.30 & 12.28 & 1.27 & 1.20 & 21.83 & 0.87 \\
\hline \multirow[t]{3}{*}[\Xi_{Q}^{\prime}\Xi_{Q}]{$_{S}^{I=1}$} & 1.25 & 0.23 & 5.31 & 0.95 & 0.80 & 2.65 \\
\hline & 1.30 & 0.47 & 4.41 & 1.00 & 1.79 & 1.97 \\
\hline & 1.40 & 1.25 & 3.08 & 1.20 & 5.95 & 1.38 \\
\hline \multirow[t]{3}{*}[\Xi_{Q}\Omega_{Q}]{$_{S}^{I=\frac{1}{2}}$} & 1.00 & 0.52 & 4.15 & 0.90 & 1.34 & 2.18 \\
\hline & 1.10 & 1.99 & 2.50 & 1.00 & 7.82 & 1.21 \\
\hline & 1.20 & 3.41 & 2.06 & 1.20 & 15.97 & 1.06 \\
\hline \multirow[t]{3}{*}[\Lambda_{Q}\Omega_{Q}]{$_{S}^{I=0}$} & 0.90 & 0.20 & 5.29 & 0.80 & 1.64 & 1.87 \\
\hline & 0.95 & 2.11 & 2.33 & 0.85 & 6.53 & 1.12 \\
\hline & 1.00 & 5.77 & 1.55 & 0.90 & 14.20 & 0.86 \\
\hline
\end{tabular}


TABLE XIII: The binding solutions of spin-triplet " $A_{Q} B_{Q}$ " systems when the interaction potential includes the contact term.

\begin{tabular}{|c|c|c|c|c|c|c|c|c|c|c|}
\hline \multirow[b]{3}{*}{ States } & \multicolumn{10}{|c|}{$S=1$} \\
\hline & \multicolumn{5}{|c|}{$Q=c$} & \multicolumn{5}{|c|}{$Q=b$} \\
\hline & $\Lambda(\mathrm{GeV})$ & B.E.(MeV) & $r_{r m s}(\mathrm{fm})$ & $P_{S}(\%)$ & $P_{D}(\%)$ & $\Lambda(\mathrm{GeV})$ & B.E.(MeV) & $r_{r m s}(\mathrm{fm})$ & $P_{S}(\%)$ & $P_{D}(\%)$ \\
\hline \multirow[t]{3}{*}[\Sigma_{Q}\Xi_{Q}^{\prime}]{$_{S}^{I=\frac{1}{2}}$} & 0.90 & 1.39 & 2.97 & 96.08 & 3.92 & 0.80 & 5.24 & 1.51 & 88.01 & 11.99 \\
\hline & 1.00 & 8.64 & 1.51 & 93.78 & 6.22 & 0.90 & 18.07 & 1.02 & 86.04 & 13.96 \\
\hline & 1.10 & 20.31 & 1.14 & 92.74 & 7.26 & 0.95 & 27.54 & 0.90 & 85.53 & 14.47 \\
\hline \multirow[t]{3}{*}[\Sigma_{Q}\Xi_{Q}^{\prime}]{$_{S}^{I=\frac{3}{2}}$} & 0.85 & 3.04 & 1.98 & 97.19 & 2.81 & 0.80 & 16.92 & 0.82 & 92.42 & 7.58 \\
\hline & 0.90 & 8.66 & 1.30 & 96.77 & 3.23 & 0.85 & 36.05 & 0.65 & 91.49 & 8.51 \\
\hline & 0.95 & 16.21 & 1.00 & 97.31 & 2.69 & 0.90 & 56.13 & 0.54 & 92.82 & 7.18 \\
\hline \multirow{3}{*}[\Xi_{Q}^{\prime}\Omega_{Q}]{$_{S}^{I=\frac{1}{2}}$} & 0.90 & 2.37 & 2.12 & 99.38 & 0.62 & 0.85 & 11.23 & 0.80 & 99.56 & 0.44 \\
\hline & 0.95 & 10.33 & 1.19 & 98.51 & 1.49 & 0.90 & 31.96 & 0.61 & 97.38 & 2.62 \\
\hline & 1.00 & 20.87 & 0.91 & 98.08 & 1.92 & 0.95 & 56.35 & 0.52 & 95.42 & 4.58 \\
\hline \multirow[t]{3}{*}[\Sigma_{Q}\Omega_{Q}]{$_{S}^{I=1}$} & 0.95 & 1.91 & 2.42 & 99.84 & 0.16 & 0.80 & 1.35 & 2.03 & 99.27 & 0.23 \\
\hline & 1.00 & 5.15 & 1.62 & 99.71 & 0.29 & 0.85 & 5.53 & 1.20 & 99.48 & 0.52 \\
\hline & 1.10 & 15.31 & 1.08 & 99.40 & 0.60 & 0.90 & 12.11 & 0.92 & 99.17 & 0.83 \\
\hline \multirow[t]{3}{*}[\Xi_{Q}\Lambda_{Q}]{$_{S}^{I=\frac{1}{2}}$} & 1.00 & 0.73 & 3.67 & 100.00 & 0.00 & 0.80 & 0.55 & 2.94 & 100.00 & 0.00 \\
\hline & 1.10 & 1.77 & 2.55 & 100.00 & 0.00 & 0.85 & 4.93 & 1.25 & 100.00 & 0.00 \\
\hline & 1.20 & 2.31 & 2.28 & 100.00 & 0.00 & 0.90 & 10.39 & 0.96 & 100.00 & 0.00 \\
\hline \multirow[t]{3}{*}[\Sigma_{Q}\Lambda_{Q}]{$_{S}^{I=1}$} & 0.80 & 2.19 & 2.50 & 95.11 & 4.98 & 0.80 & 28.86 & 0.79 & 87.95 & 12.05 \\
\hline & 0.85 & 5.32 & 1.75 & 95.23 & 4.77 & 0.85 & 41.93 & 0.67 & 89.83 & 10.17 \\
\hline & 0.90 & 9.34 & 1.39 & 95.67 & 4.33 & 0.90 & 56.53 & 0.58 & 92.07 & 7.93 \\
\hline \multirow[t]{3}{*}[\Sigma_{Q}\Xi_{Q}]{$_{S}^{I=\frac{1}{2}}$} & 0.90 & 0.57 & 3.99 & 99.96 & 0.04 & 0.80 & 1.10 & 2.22 & 99.12 & 0.08 \\
\hline & 1.00 & 9.52 & 1.31 & 99.88 & 0.12 & 0.85 & 6.44 & 1.15 & 99.72 & 0.28 \\
\hline & 1.10 & 25.78 & 0.92 & 99.88 & 0.12 & 0.90 & 15.96 & 0.84 & 99.56 & 0.44 \\
\hline \multirow[t]{3}{*}[\Sigma_{Q}\Xi_{Q}]{$_{S}^{I=\frac{3}{2}}$} & 0.95 & 0.49 & 4.16 & 99.81 & 0.19 & 0.80 & 4.04 & 1.29 & 99.59 & 0.41 \\
\hline & 1.00 & 1.56 & 2.60 & 99.68 & 0.32 & 0.85 & 0.90 & 0.98 & 98.73 & 1.27 \\
\hline & 1.10 & 6.02 & 1.45 & 99.61 & 0.39 & 0.90 & 14.91 & 0.82 & 97.99 & 2.01 \\
\hline \multirow[t]{3}{*}[\Xi_{Q}^{\prime}\Lambda_{Q}]{$_{S}^{I=\frac{1}{2}}$} & 0.95 & 1.25 & 2.91 & 99.88 & 0.12 & 0.80 & 3.15 & 1.43 & 99.76 & 0.24 \\
\hline & 1.00 & 3.64 & 1.85 & 99.83 & 0.17 & 0.85 & 8.40 & 1.01 & 99.40 & 0.60 \\
\hline & 1.10 & 12.23 & 1.14 & 99.83 & 0.17 & 0.90 & 15.51 & 0.82 & 99.15 & 0.85 \\
\hline \multirow[t]{3}{*}[\Xi_{Q}^{\prime}\Xi_{Q}]{$_{S}^{I=0}$} & 0.90 & 4.89 & 1.67 & 98.42 & 1.58 & 0.80 & 5.71 & 1.39 & 86.06 & 13.94 \\
\hline & 0.95 & 14.60 & 1.10 & 98.93 & 1.07 & 0.85 & 16.62 & 0.90 & 91.74 & 8.26 \\
\hline & 1.00 & 28.43 & 0.86 & 99.36 & 0.64 & 0.90 & 35.81 & 0.66 & 96.05 & 3.95 \\
\hline \multirow[t]{3}{*}[\Xi_{Q}^{\prime}\Xi_{Q}]{$_{S}^{I=1}$} & 0.90 & 0.81 & 3.41 & 99.63 & 0.37 & 0.80 & 0.77 & 2.50 & 98.86 & 1.14 \\
\hline & 0.95 & 4.39 & 1.69 & 99.39 & 0.61 & 0.85 & 10.07 & 0.93 & 98.57 & 1.43 \\
\hline & 1.00 & 9.88 & 1.22 & 99.31 & 0.69 & 0.90 & 23.63 & 0.70 & 98.19 & 1.81 \\
\hline \multirow[t]{3}{*}[\Xi_{Q}\Omega_{Q}]{$_{S}^{I=\frac{1}{2}}$} & 0.90 & 0.45 & 4.22 & 99.75 & 0.25 & 0.85 & 3.01 & 1.45 & 97.79 & 2.21 \\
\hline & 0.95 & 5.94 & 1.49 & 99.34 & 0.66 & 0.90 & 19.44 & 0.76 & 97.00 & 3.00 \\
\hline & 1.00 & 15.35 & 1.04 & 99.23 & 0.77 & 0.95 & 41.92 & 0.58 & 97.13 & 2.87 \\
\hline \multirow[t]{3}{*}[\Lambda_{Q}\Omega_{Q}]{$_{S}^{I=0}$} & 0.90 & 0.20 & 5.29 & 100.00 & 0.00 & 0.80 & 1.64 & 1.87 & 100.00 & 0.00 \\
\hline & 0.95 & 2.11 & 2.33 & 100.00 & 0.00 & 0.85 & 6.53 & 1.12 & 100.00 & 0.00 \\
\hline & 1.00 & 5.77 & 1.55 & 100.00 & 0.00 & 0.90 & 14.20 & 0.86 & 100.00 & 0.00 \\
\hline
\end{tabular}




\section{The Numerical Results of The Baryon-antibaryon Systems With the OPE potential}

As a byproduct, we present the binding solutions of the heavy baryon-antibaryon systems with the pion-exchange potential. It is straightforward to obtain the potential via changing the sign of the potential for the baryon-baryon systems since the G-parity of the pion is negative. Our results indicate that the pion-exchange alone is strong enough to form some bound states. The numerical results are collected in Tables XIV XV

TABLE XIV: The numerical results of the spin-singlet heavy baryon-antibaryon systems with the OPE potential. " $\Lambda$ " is the cutoff parameter. "B.E." is the binding energy, and " $r_{r m s}$ " is the root-mean-square radius which reflects the size of the bound state. " $\times$ " denotes no binding solutions

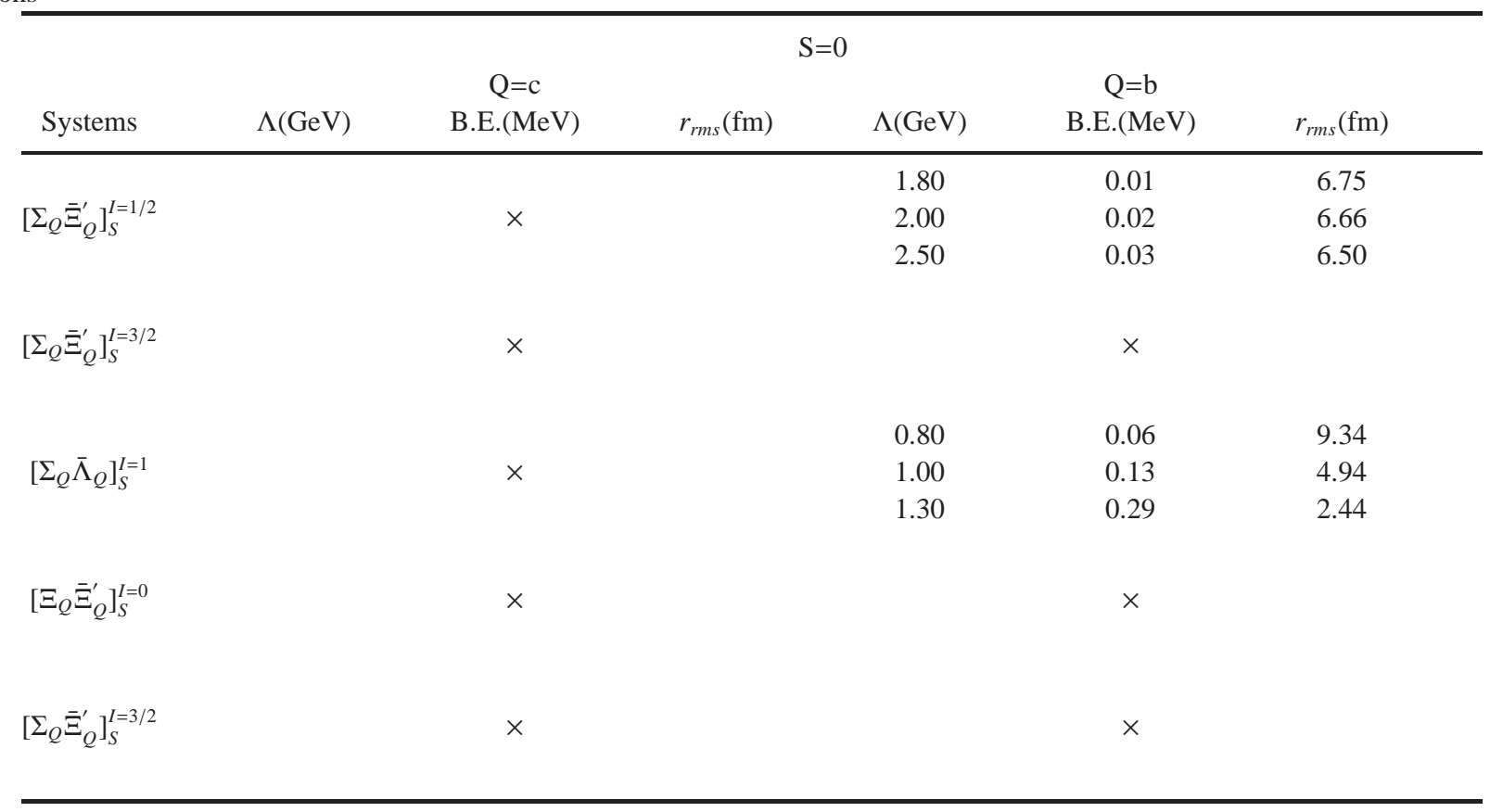

D. The Dependence of The Binding Energy on The Cutoff Parameter 
TABLE XV: The numerical results of the spin-triplet heavy baryon-antibaryon systems with the OPE potential. " $\Lambda$ " is the cutoff parameter. "B.E." is the binding energy while " $r_{r m s}$ " is the root-mean-square radius. " $P_{S}$ " and " $P_{D}$ " are the probabilities of the $\mathrm{S}$ wave and the $\mathrm{D}$ wave, respectively. " $x$ " denotes no binding solutions.

\begin{tabular}{|c|c|c|c|c|c|c|c|c|c|c|}
\hline Systems & $\Lambda(\mathrm{GeV})$ & B.E.(MeV) & $r_{r m s}(\mathrm{fm})$ & $P_{S}$ & $P_{D}$ & $\Lambda(\mathrm{GeV})$ & B.E.(MeV) & $r_{r m s}(\mathrm{fm})$ & $P_{S}(\%)$ & $P_{D}(\%)$ \\
\hline \multirow{3}{*}[\Sigma_{Q}\overline{\Xi}_{Q}^{\prime}]{$_{S}^{I=1 / 2}$} & 1.20 & 1.37 & 2.80 & 89.79 & 10.21 & 0.80 & 4.05 & 1.60 & 74.87 & 25.13 \\
\hline & 1.30 & 4.95 & 1.69 & 84.74 & 15.26 & 0.90 & 9.86 & 1.21 & 70.37 & 29.63 \\
\hline & 1.40 & 11.24 & 1.25 & 81.80 & 18.92 & 1.00 & 19.09 & 0.98 & 67.15 & 32.85 \\
\hline \multirow{3}{*}[\Sigma_{Q}\overline{\Xi}_{Q}^{\prime}]{$_{S}^{I=3 / 2}$} & & & & & & 1.20 & 0.72 & 2.68 & 94.08 & 5.92 \\
\hline & & & $\times$ & & & 1.30 & 1.75 & 1.88 & 92.19 & 7.81 \\
\hline & & & & & & 1.50 & 5.80 & 1.18 & 89.44 & 10.56 \\
\hline$\left[\Sigma_{Q} \bar{\Lambda}_{Q}\right]_{S}^{I=1}$ & 1.60 & 0.03 & 6.65 & 97.47 & 2.53 & 0.90 & 1.41 & 2.75 & 87.92 & 12.08 \\
\hline \multirow[t]{2}{*}[\Xi_{Q}\overline{\Xi}_{Q}^{\prime}]{$_{S}^{I=0}$} & 2.30 & 3.16 & 2.89 & 94.51 & 5.49 & 1.20 & 1.89 & 1.91 & 90.07 & 9.93 \\
\hline & 2.50 & 9.15 & 1.20 & 92.55 & 7.45 & 1.40 & 6.22 & 1.20 & 87.85 & 12.15 \\
\hline$\left[\Xi_{Q} \bar{\Xi}_{Q}^{\prime}\right]_{S}^{I=1}$ & & & $\times$ & & & & & $\times$ & & \\
\hline
\end{tabular}
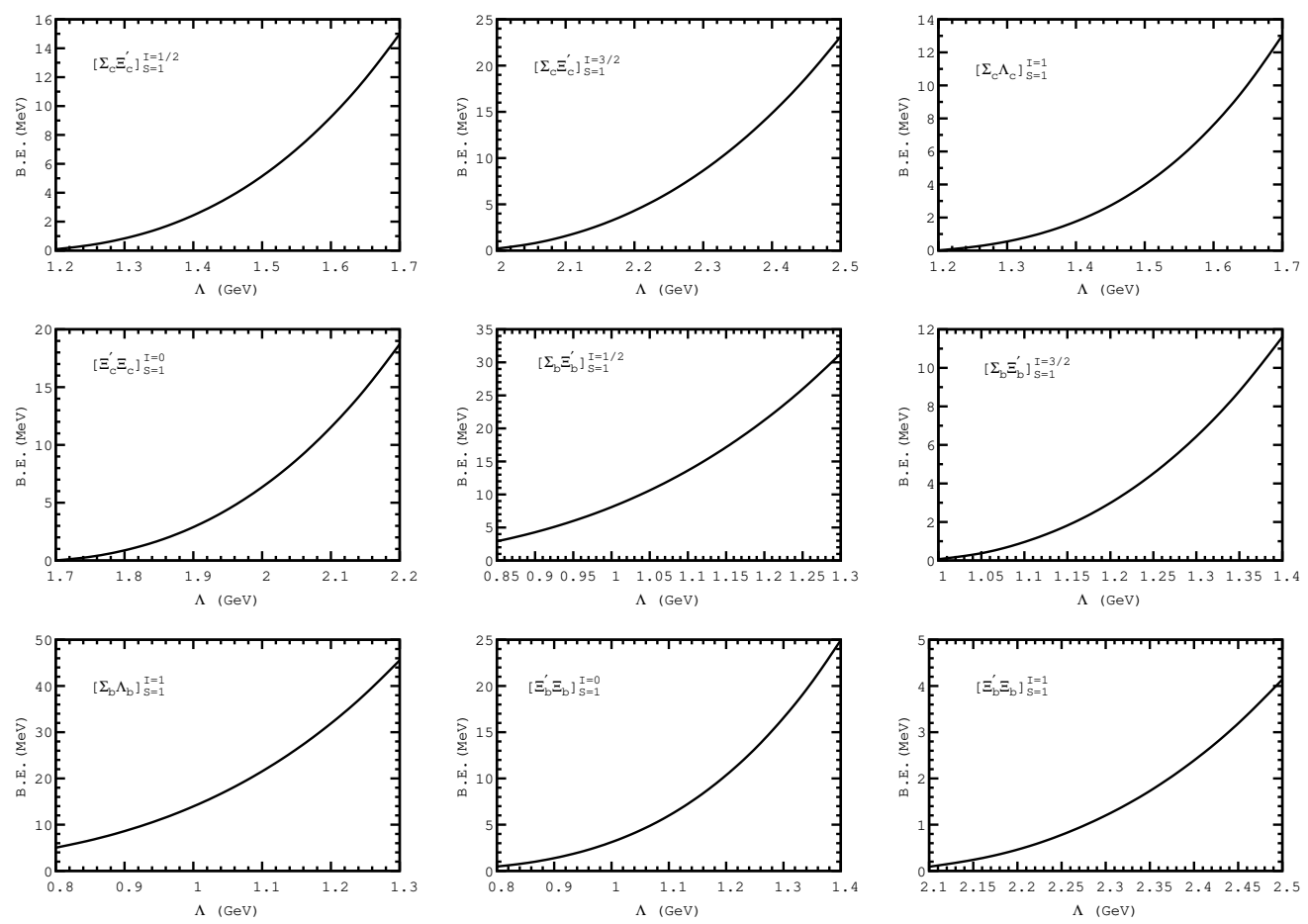

FIG. 5: The dependence of the binding energy on the cutoff parameter for the " $A_{Q} B_{Q}$ " system with the OPE potential. 

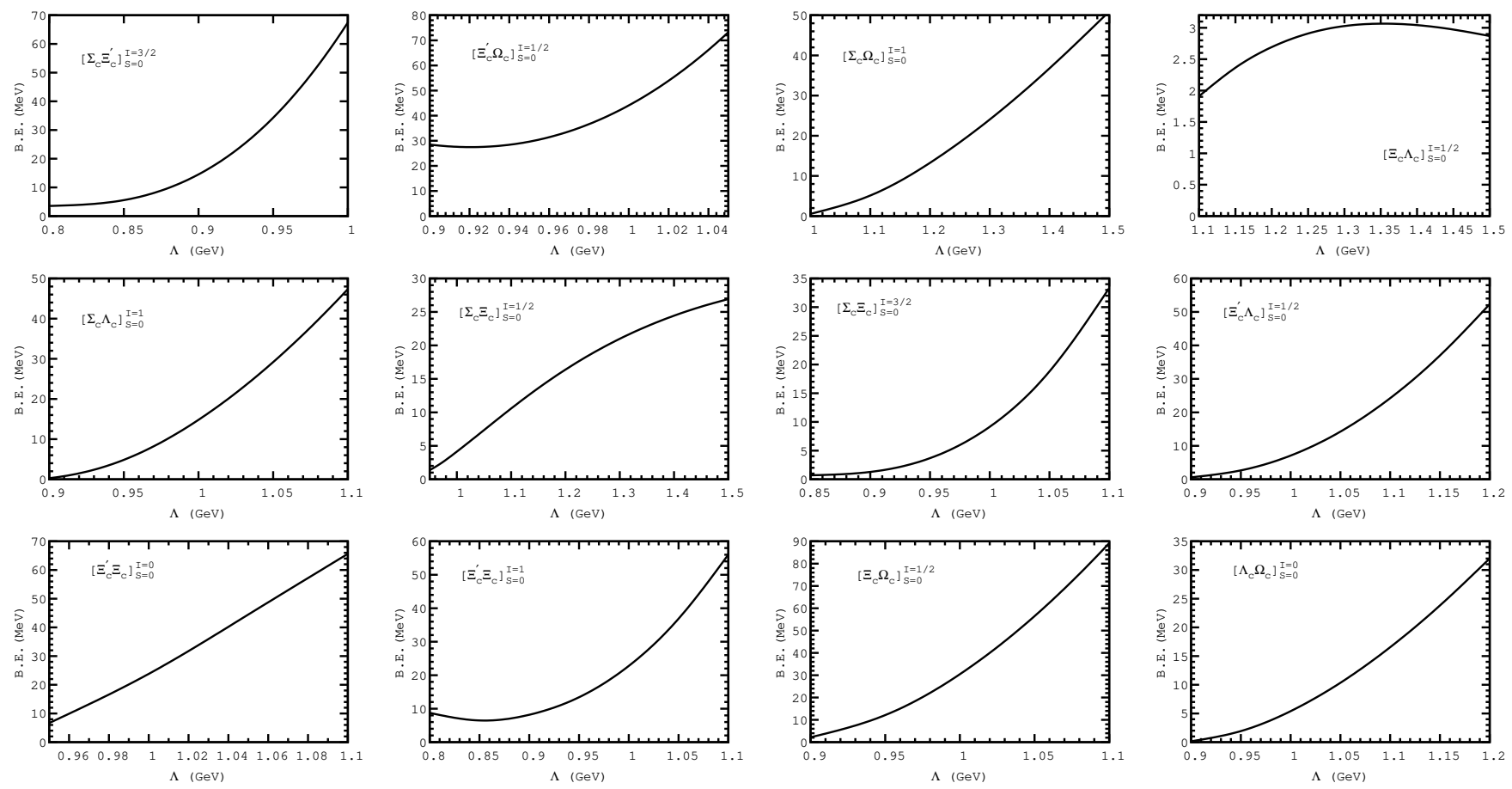

FIG. 6: The dependence of the binding energy on the cutoff momentum for the spin-singlet system " $A_{c} B_{c}$ " with the OBE potential.
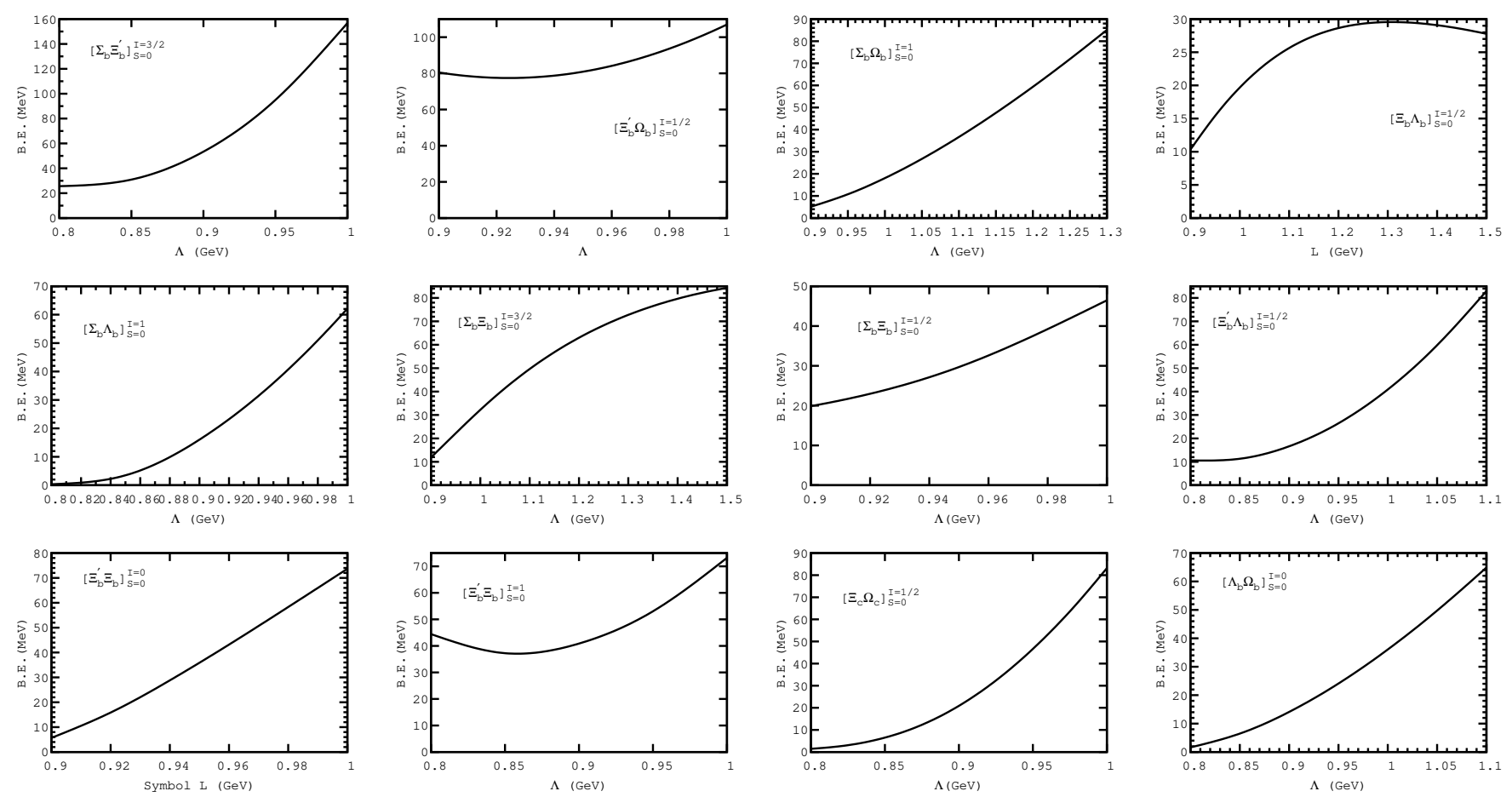

FIG. 7: The dependence of the binding energy on the cutoff parameter for the spin-singlet " $A_{b} B_{b}$ " system with OBE potential. 

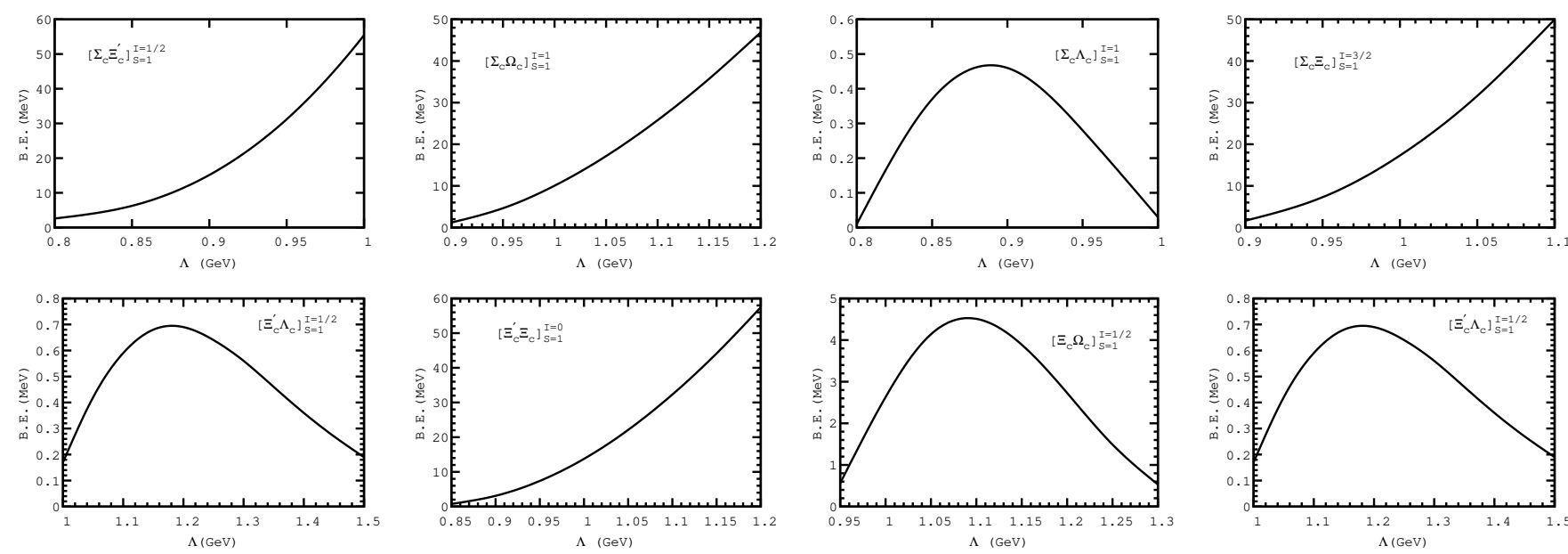

FIG. 8: The dependence of the binding energy on the cutoff parameter for the spin-triplet " $A_{c} B_{c}$ " system with the OBE potential.
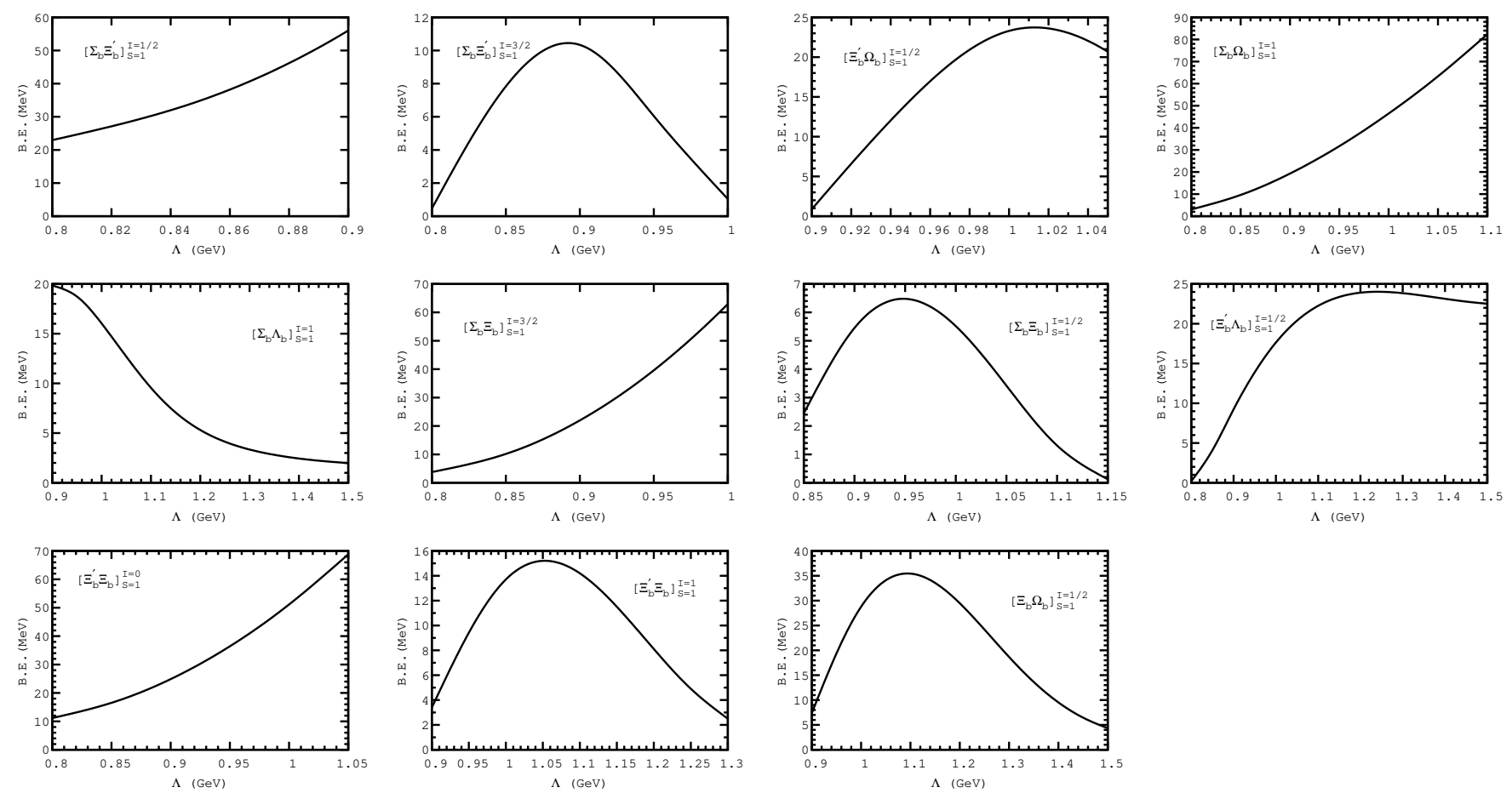

FIG. 9: The dependence of the binding energy on the cutoff parameter for the spin-triplet " $A_{b} B_{b}$ " system with the OBE potential. 

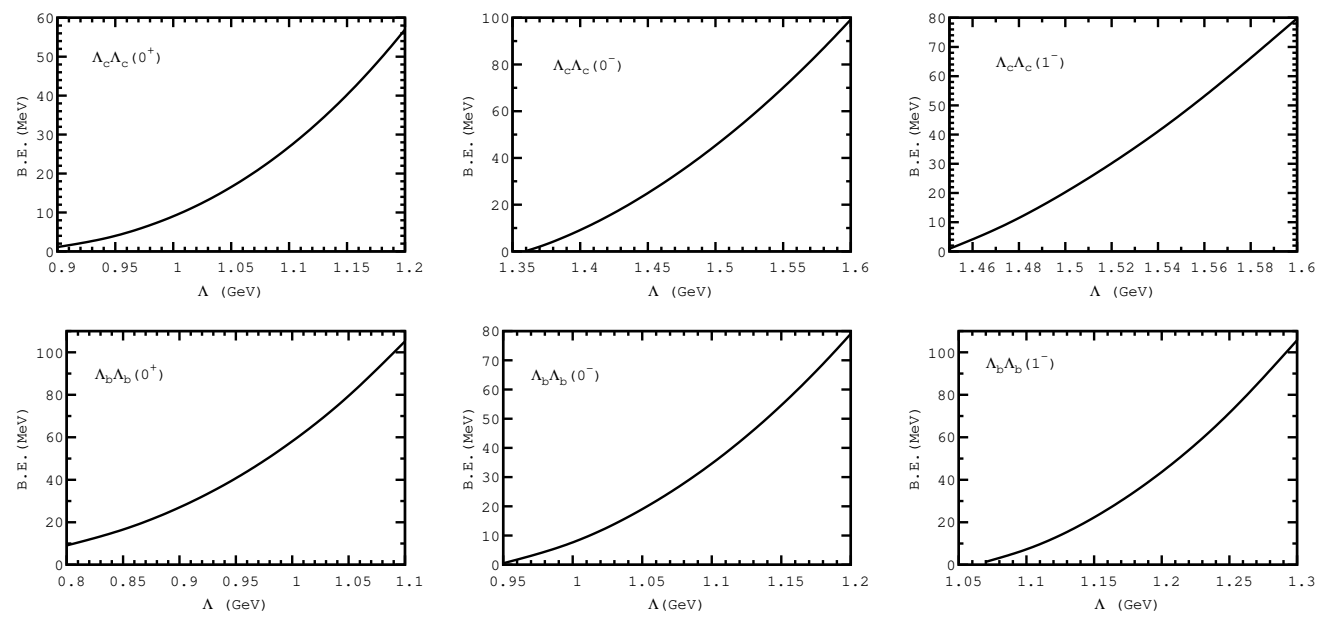

FIG. 10: The dependence of the binding energy for $\Lambda_{Q} \Lambda_{Q}$ on the cutoff parameter with the OPE potential. 